\title{
Clocking the assembly of double-barred galaxies with the MUSE TIMER project
}

\author{
Adriana de Lorenzo-Cáceres ${ }^{\circledR},{ }^{1,2 \star}$ Patricia Sánchez-Blázquez, ${ }^{3}$ \\ Jairo Méndez-Abreu ${ }^{\ominus},{ }^{1,2}$ Dimitri A. Gadotti, ${ }^{4}$ Jesús Falcón-Barroso, ${ }^{1,2}$ \\ Inma Martínez-Valpuesta, ${ }^{1,2}$ Paula Coelho, ${ }^{5}$ Francesca Fragkoudi, ${ }^{6}$ \\ Bernd Husemann ${ }^{\oplus} 7$ Ryan Leaman, ${ }^{7}$ Isabel Pérez, ${ }^{8,9}$ Miguel Querejeta, ${ }^{4,10}$ \\ Marja Seidel $^{11,12}$ and Glenn van de Ven ${ }^{\odot 4,7}$ \\ ${ }^{1}$ Instituto de Astrofísica de Canarias, Calle Vía Láctea s/n, E-38205 La Laguna, Tenerife, Spain \\ ${ }^{2}$ Departamento de Astrofísica, Universidad de La Laguna, E-38200 La Laguna, Tenerife, Spain \\ ${ }^{3}$ Departamento de Física Teórica, Universidad Autónoma de Madrid, E-28049 Cantoblanco, Spain \\ ${ }^{4}$ European Southern Observatory, Karl-Schwarzschild-Str. 2, D-85748 Garching bei München, Germany \\ ${ }^{5}$ Instituto de Astronomia, Geofísica e Ciencias Atmosféricas, Universidade de Sao Paulo, Rua do Matao, 1226, 05508-090 Sao Paulo-SP, Brazil \\ ${ }^{6}$ Max-Planck-Institut fur Astrophysik, Karl-Schwarzschild-Str. 1, D-85748 Garching bei München, Germany \\ ${ }^{7}$ Max-Planck-Institut fur Astronomie, Konigstuhl 17, D-69117 Heidelberg, Germany \\ ${ }^{8}$ Departamento de Física Teórica y del Cosmos, Universidad de Granada, Facultad de Ciencias (Edificio Mecenas), E-18071 Granada, Spain \\ ${ }^{9}$ Instituto Universitario Carlos I de Física Teórica y Computacional, Universidad de Granada, E-18071 Granada, Spain \\ ${ }^{10}$ Observatorio Astronómico Nacional (IGN), C/ Alfonso XII 3, E-28014 Madrid, Spain \\ ${ }^{11}$ Caltech-IPAC, Spitzer Science Center, 1200 E. California Blvd., Pasadena, CA 91125, USA \\ ${ }^{12}$ The Observatories of the Carnegie Institution for Science, 813 Santa Barbara St., Pasadena, CA 91101, USA
}

Accepted 2019 January 17. Received 2019 January 15; in original form 2018 September 7

\begin{abstract}
The formation of two stellar bars within a galaxy has proved challenging for numerical studies. It is not yet clear whether the inner bar is born via a star formation process promoted by gas inflow along the outer bar or whether it is dynamically assembled from instabilities in a small-scale stellar disc. Observational constraints to these scenarios are scarce. We present a thorough study of the stellar content of two double-barred galaxies observed by the MUSE TIMER project, NGC 1291 and NGC 5850, combined with a two-dimensional multicomponent photometric decomposition performed on the $3.6 \mu \mathrm{m}$ images from $\mathrm{S}^{4} \mathrm{G}$. Our analysis confirms the presence of $\sigma$-hollows appearing in the stellar velocity dispersion distribution at the ends of the inner bars. Both galaxies host inner discs matching in size with the inner bars, suggestive of a dynamical formation for the inner bars from small-scale discs. The analysis of the star formation histories for the structural components shaping the galaxies provides constraints on the epoch of dynamical assembly of the inner bars, which took place $>6.5 \mathrm{Gyr}$ ago for NGC 1291 and >4.5 Gyr ago for NGC 5850. This implies that inner bars are long-lived structures.
\end{abstract}

Key words: galaxies: evolution-galaxies: individual: NGC 1291-galaxies: individual: NGC 5850 - galaxies: kinematics and dynamics - galaxies: stellar content - galaxies: structure.

\section{INTRODUCTION}

Double-barred galaxies are disc galaxies that host two embedded, non-axisymmetric, bar-shaped stellar structures. The outer bar is the regular stellar bar found in 60-70 per cent of all disc galaxies (Marinova \& Jogee 2007; Aguerri, Méndez-Abreu \& Corsini 2009;

^E-mail: adrianadelorenzocaceres@gmail.com
Méndez-Abreu et al. 2012, 2017, among others), which appears alone in the majority of the galaxies. The smaller inner bar is sometimes referred to as a 'nuclear bar' or 'secondary bar', although we prefer avoiding the use of these terms as they hold implications for either its size or its formation process that have not been proven yet, as explained below. The inner bar appears randomly oriented with respect to the outer bar due to their different pattern speeds, as found in both simulations and observations (Friedli \& Martinet 1993; Corsini, Debattista \& Aguerri 2003). 
Current estimates indicate that 30 per cent of all early-type barred galaxies are actually double-barred (Erwin \& Sparke 2002; Laine et al. 2002; Erwin 2004). This fraction may even be considered a lower limit: Since inner-bar detection has traditionally relied on direct (Buta et al. 2015) or indirect (ellipse fitting, unsharp masking; Erwin 2004) photometric identification, it is hampered by the overlapping of many structural components (bulges, inner discs, underlying outer bar and disc) in the central galaxy regions where the inner bar is embedded. Indeed, this fact together with the presence of dust has prevented the photometric search for doublebarred systems in the latest galaxy types (beyond Sb; Erwin 2005, 2011).

Outer bars are able to promote secular evolution in a significant way, thanks to their ability of transporting material (gas and stars) inwards and outwards (e.g. Muñoz-Tuñón, Caon \& Aguerri 2004; Sheth et al. 2005). Inner bars have been proposed to contribute to gas inflow as well, even reaching the regions of influence of the central black holes and igniting nuclear activity (active galactic nuclei, AGNs; Shlosman, Frank \& Begelman 1989; Shlosman, Begelman \& Frank 1990). Although signatures of gas flow from the outer to the inner bars have actually been detected in double-barred galaxies (de Lorenzo-Cáceres et al. 2012; de Lorenzo-Cáceres, Falcón-Barroso \& Vazdekis 2013), no clear evidence of associated star-forming regions or newly formed structures due to inner bars has yet been found. The long-lived nature of inner bars is also under debate.

The two most extensive catalogues of double-barred galaxies to date are those presented by Erwin (2004) and Buta et al. (2015). Erwin (2004) uses a compilation of images from different sources, including some Hubble Space Telescope (HST) data, and identifies 50 double-barred galaxies. Buta et al. (2015) perform the morphological classification of over 2000 galaxies from the Spitzer Survey of Stellar Structure in Galaxies $\left(S^{4} \mathrm{G}\right.$; Sheth et al. 2010), finding 15 double-barred galaxies. Both studies have nine individuals in common. We note however that some inner bars are misclassified with other central non-barred components, such as inner rings, inner discs, inner spirals, or star-forming spots that overlap and resemble elongated components when observed with limited spatial resolution. In particular, the mean point-spread function (PSF) of the $\mathrm{S}^{4} \mathrm{G} 3.6 \mu \mathrm{m}$ data is 1.66 arcsec.

Inner bars typically extend from 0.3 up to $2.5 \mathrm{kpc}$ in length (projected semimajor axes; de Lorenzo-Cáceres et al. 2019b). These numbers have been for the first time measured with two-dimensional multicomponent photometric decompositions and they reveal that inner bars may be long structures, far from the nuclear scales of less than $1 \mathrm{kpc}$ in full length previously considered (Erwin 2011). Note moreover that inner bars can be as large as some of the smallest single bars (Erwin 2005). Notwithstanding, these measurements still mean inner bars can be as short as just a couple of arcseconds even at the nearby distances of several known double-barred galaxies. Spatial resolution is therefore key when detecting and analysing inner bars and it explains the small amount of double-barred systems detected within $\mathrm{S}^{4} \mathrm{G}$.

Observational spectroscopic studies of double-barred galaxies are scarce. First approaches focused on the analysis of their stellar kinematics with long-slit (Emsellem et al. 2001) and integral-field spectra (Moiseev, Valdés \& Chavushyan 2004). This last technique soon proved the best strategy to study structurally complex objects such as double-barred galaxies. The suite of works by de Lorenzo-Cáceres et al. (2008, 2012, 2013), based on long-slit and integral-field data from the EMMI@NTT and SAURON@WHT spectrographs, respectively, represents the most extensive analysis of not only the stellar kinematics but also the stellar populations and ionized gas content of double-barred galaxies.

de Lorenzo-Cáceres et al. (2008) found that the main kinematic signature caused by inner bars in the line-of-sight velocity distribution (LOSVD) affects the velocity dispersion. Indeed, while the distortion of the velocity field induced by the inner bar is very subtle (a slight double-hump profile predicted by the simulations of Bureau \& Athanassoula 2005), the spatial distribution of the velocity dispersion shows the so-called $\sigma$-hollows: two local minima located at the ends of the inner bars. de Lorenzo-Cáceres et al. (2008) proposed the $\sigma$-hollows appear due to a contrast effect between the high velocity dispersion of the dynamically hotter central bulge and the cooler inner bar. At the bar ends, where this structure starts dominating the galaxy light over the bright bulge, the lower $\sigma$ values become evident. More recently, numerical simulations by Du et al. (2017) found that $\sigma$-hollows may be a physical property of all, even single, bars, particularly related to the vertical component of the velocity dispersion of the disc where the bar is developed. The amplitude of the hollows is stronger for shorter bars, which explains why they have been observed only in inner bars within double-barred systems. Why the vertical component of $\sigma$ would be affected this way is still unknown. We note here that $\sigma$-hollows have been observed in all the double-barred galaxies whose stellar kinematics has been studied to date (see e.g. de Lorenzo-Cáceres et al. 2012; de Lorenzo-Cáceres et al. 2013; Du et al. 2016).

The study of the formation and evolution of double-barred galaxies with numerical simulations has proved particularly challenging. How single bars are created from instabilities in dynamically cold discs is a relatively well-understood process (e.g. Combes et al. 1990; Debattista \& Sellwood 2000; Athanassoula 2003). However, obtaining two coexistent bars in a simulated disc galaxy is far more complicated. Most of the effort in this direction can be classified within two main scenarios.

The first case (hereafter scenario 1) includes the works by Friedli \& Martinet (1993), Heller, Shlosman \& Englmaier 2001, Shlosman \& Heller 2002, and Englmaier \& Shlosman 2004, among others, where a gaseous and eventually a stellar inner bar is directly formed after gas inflow through the outer bar. This gas is trapped in the outer-bar $x_{2}$ orbits and therefore the prior formation of the largescale bar is a requirement. Since they are born from star formation triggered by gas collapse, inner bars formed this way are expected to be younger than outer bars (with the magnitude of the differences depending on the time-scale of the whole formation process). Such prediction has actually been measured in observations, particularly in the spectroscopic analysis of de Lorenzo-Cáceres et al. (2013). The simulations corresponding to this scenario find that inner bars are transient structures which dissolve very quickly (in a few hundreds of Myr) since they do not have the dynamical support of e.g. a cooler structure. However, the large fraction of inner bars found in nature and the fact that inner bars have been observed up to a redshift $z \sim 0.15$ (Lisker et al. 2006) suggest they live for long periods or, if transient, that they are successively destroyed and reformed on short time-scales.

The second path (scenario 2) for double-barred formation poses that inner bars form dynamically from inner discs, in the same way as large-scale bars do, but at a smaller spatial scale. This represents a collisionless way of forming double-barred systems, as the inner bar formation happens dynamically out of stars and does not involve star formation. After first attempts by Friedli \& Martinet (1993), Rautiainen \& Salo (2000), and Rautiainen, Salo \& Laurikainen (2002), the works by Debattista \& Shen (2007), Shen \& Debattista (2009), and more recently Du, Shen \& Debattista (2015) 
successfully managed to create stable inner bars without invoking the presence of gas. They used pure $N$-body simulations in which they placed a small disc-like structure, which can be named a disclike bulge or inner disc, within a disc galaxy. Although the smallscale bar developed faster from the inner disc than the large-scale bar from the main disc, the authors argued that small-scale discs are usually formed through secular evolution promoted by the main bar. Outer bars are most likely formed first and, although this is not a requirement in this scenario, it is in full agreement with the recent formation process proposed by Wozniak (2015). This author develops an inner bar via a stellar inner disc that is formed through gas inflow along the previously formed outer bar.

Within this second scenario, there are no strict constraints on the relative ages between inner and outer bars. They may both be coeval, as in the case of the double-barred galaxy NGC 357 (de LorenzoCáceres et al. 2012). However, younger and more metal-rich inner bars, as those observed by de Lorenzo-Cáceres et al. (2013) and mentioned before, are also in agreement, depending on the amount of gas present and the time-scales for star formation.

One of the main differences between scenario 2 and scenario 1 is that inner bars simulated this way can be long-lived. For example, Wozniak (2015) forms inner bars that live for up to $7 \mathrm{Gyr}$. Disentangling the nature of inner bars is very important since longlived inner bars can be considered serious candidates for explaining the long-debated problem of black hole feeding.

Nowadays it is clear that spectroscopic observations and a careful analysis of the structures inhabiting the central regions of doublebarred galaxies are needed to recover their formation histories and finally constrain the possible formation scenarios. Our first efforts in de Lorenzo-Cáceres et al. $(2012,2013)$ were pioneering in this field. Following these steps, in this paper we present an analysis of the stellar kinematics and stellar populations of the Time Inference with MUSE in Extragalactic Rings (TIMER; Gadotti et al. 2019, hereafter Paper I) double-barred galaxies, which allows us to study their properties with unprecedented detail thanks to the high spatial resolution and large field-of-view (FoV) provided by MUSE. We combine an analysis of the star formation histories (SFHs) with the structural information provided by two-dimensional (2D) multicomponent photometric decompositions of the sample galaxies. This combination allows us to constrain the epoch of assembly of the different structural components of double-barred galaxies for the first time. While we focus here on the stellar content, the properties of the ionized gas content and gas kinematics in double-barred galaxies are also within the goals of TIMER and will be presented elsewhere.

A summary of the design, observations, and goals of the TIMER project is provided in Section 2, together with a description of the TIMER double-barred galaxies. Sections 3, 4, and 5 describe the analysis of their photometric decompositions, stellar kinematics, and stellar populations, respectively. Discussion of the most notable stellar kinematic features and the formation scenario of doublebarred galaxies is presented in Section 6. Finally, in Section 7 we report on the main results and conclusions of this work.

\section{THE TIMER DATA SET}

The Time Inference with MUSE in Extragalactic Rings project is a survey with the VLT-MUSE integral-field spectrograph of 24 nearby barred galaxies with prominent central structures, such as nuclear rings, inner spiral arms, inner bars, and inner discs (see Paper I). One of the project's main goals is to study the SFH of such structures to infer the cosmic epoch of the formation of the bar and the dynamical settling of the main disc. The methodology was demonstrated with a pilot study of NGC 4371 (Gadotti et al. 2015).

The TIMER sample was drawn from the $S^{4} \mathrm{G}$ (Sheth et al. 2010), which includes only galaxies at distances below $40 \mathrm{Mpc}$, brighter than 15.5 B-mag, and larger than 1 arcmin. The TIMER galaxies are all barred, with stellar masses above $10^{10} \mathrm{M}_{\odot}$ and inclinations below $\sim 60^{\circ}$. The presence of the bar and inner structures was assessed from the morphological classifications of Buta et al. (2015).

Most of the observations were performed during ESO Period 97 (2016 April to September) with a typical seeing of $0.8-0.9$ arcsec, mean spectral resolution of $2.65 \AA$ (full width at half-maximum, FWHM), and spectral coverage from $4750 \AA$ to $9350 \AA$. MUSE covers an almost square $1 \times 1 \operatorname{arcmin}^{2} \mathrm{FoV}$ with a contiguous spatial sampling of $0.2 \times 0.2 \operatorname{arcsec}^{2}$ and a spectral sampling of $1.25 \AA$ per pixel.

The MUSE pipeline (version 1.6) was used to reduce the data set (bias, flat-fielding, wavelength and flux calibrations, sky; Weilbacher et al. 2012). We refer the reader to Paper I for further details on the sample selection, observations, and data reduction.

\subsection{TIMER double-barred galaxies}

Among the full TIMER sample, 7 out of 24 galaxies have been previously reported to host double bars either by Erwin (2004; 7 galaxies) or by Buta et al. (2015; 4 galaxies). NGC 1291, NGC 1433, NGC 5728, and NGC 5850 are the four double-barred galaxies as classified in both studies, while Erwin (2004) includes also NGC 1097, NGC 4303, and NGC 4984 in his catalogue. We note, however, that some of these inner bars may have been misclassified due to the presence of other central structures such as inner discs, inner spirals, inner rings, and star-forming regions. This is actually the case for the following galaxies, for which we have inspected recent HST images in bandfilters covered by the TIMER spectral range: NGC 1097 (proposal ID: 13413; PI: K. Sheth), NGC 1433 and NGC 4303 (proposal ID: 9042; PI: S. Smartt), NGC 4984 (proposal ID: 15133; PI: P. Erwin, private communication), and NGC 5728 (proposal ID: 13755; PI: J. Greene).

NGC 1291 and NGC 5850 are undoubtedly double-barred hosts. NGC 1291 is a close-by (distance of $8.6 \mathrm{Mpc}$ ) and almost face-on ( $i$ $\sim 11^{\circ}$ ) galaxy, whose inner bar is perfectly observed in direct images without the need for any further analysis (e.g. ellipse fitting or unsharp masking). NGC 5850 (distance $23.1 \mathrm{Mpc} ; i \sim 39^{\circ}$ ) belongs to the sample of double-barred galaxies analysed by de LorenzoCáceres et al. (2008, 2013), who confirmed the presence of the inner bar not only photometrically but also spectroscopically through the kinematic diagnostics of the $\sigma$-hollows (see Section 4). Buta et al. (2015) classified these two galaxies as (R)SAB(l,bl,nb) $0^{+}$and (R')SB(r,bl,nr,nb)ab, respectively. NGC 1291 and NGC 5850 are used throughout this paper as benchmarks to study the properties of double-barred galaxies with the superb spatial resolution provided by MUSE.

\section{TWO-DIMENSIONAL MULTICOMPONENT PHOTOMETRIC DECOMPOSITIONS}

While it would be ideal to perform the photometric decompositions using the MUSE TIMER data cubes, these spatially cover the central regions only and do not reach the galaxy disc, whose modelling is required for a good decomposition of the central structures. Given the fact that all TIMER galaxies are nearby, the spatial resolution of the $\mathrm{S}^{4} \mathrm{G}$ images is good enough to derive the extent of the central structures, including the inner bars. More importantly, $S^{4} \mathrm{G}$ images 
represent a homogeneous data set for the whole TIMER sample and the $3.6 \mu \mathrm{m}$ bandpass has the advantage of being less affected by dust effects than optical ones, which is particularly important at the galaxy centres.

The multicomponent photometric decomposition of the $3.6 \mu \mathrm{m}$ images has been carried out using the GASP2D code (Méndez-Abreu et al. 2008a, 2014). Detailed information about this analysis for the whole TIMER sample will be described in Méndez-Abreu et al. (in preparation). Here we outline the basic steps and the specifics for the two galaxies in this paper: NGC 1291 and NGC 5850.

GASP2D works in two dimensions fitting the raw counts of the images to a combination of analytical models describing the surfacebrightness distribution of the galaxy components. The non-linear fit is carried out minimizing the $\chi^{2}$ using a Levenberg-Marquardt algorithm. The models are convolved with the image PSF before the $\chi^{2}$ is computed in each iteration. The $S^{4} \mathrm{G} 3.6 \mu \mathrm{m}$ PSF was assumed to be well described with a Moffat function, whose FWHM and shape parameter $(\beta)$ were computed as the mean of several stars in each image (the mathematical description of the Moffat function can be found in e.g. Méndez-Abreu et al. 2008a). We found FWHM values of 1.8 and 1.9 arcsec and $\beta$ values of 2.5 and 3.0 for NGC 1291 and NGC 5850, respectively. For the fitting, each pixel was weighted according to the values provided in the weighting images supplied by the $S^{4} G$ team. Individual masks were created for each galaxy in order to avoid foreground stars or strong star-forming regions that might bias the results of the decomposition. This includes the possible outer and inner rings present in the images that were not modelled in the photometric decomposition (see below).

The final number of components used for each galaxy was decided in a bottom-up fashion and after a careful revision of both the 2D residuals (model-image) and the one-dimensional (1D) azimuthally averaged radial profiles of the surface brightness, ellipticity, and position angle (see Figs 1 and 2). Since all galaxies in the TIMER sample host a clear bar, the initial fit was performed using a bulge, a disc, and a bar component described with a Sérsic (Sérsic 1968), an exponential (Freeman 1970), and a Ferrers (Ferrers 1877) profile, respectively. The inner bars present in NGC 1291 and NGC 5850 stand out clearly in the images and therefore they were also modelled using an additional Ferrers profile. A complete description of the adopted functions for double-barred galaxies is detailed in de Lorenzo-Cáceres et al. (2019a). It is worth noting that the Ferrers profiles are implemented into a reference frame of generalized ellipses, following the description given in Athanassoula et al. (1990). Finally, we included an inner disc component (described with a Sérsic profile) in the case of NGC 5850, and both an inner disc and an outer lens (each described with a Sérsic profile) in the case of NGC 1291. In summary, we fit 6 and 5 structural components to the surface-brightness distributions of NGC 1291 and NGC 5850, respectively. The residuals inside the region corresponding to the MUSE FoV represent only 3 per cent and 6 per cent of the total flux for NGC 1291 and NGC 5850, respectively, with 99.5 per cent of the pixels having relative errors $<15$ per cent. These numbers support the robustness of our photometric decompositions to describe the structural compositions of both galaxies within the region under study.

Our strategy of building up successively more complex models starting from relatively simple ones was successfully tested in Méndez-Abreu et al. (2017), and it allows us to gradually fix some parameters and reduce the highly degenerate parameter space. The results from our 2D photometric decompositions are shown in Table 1 and in Figs 1 and 2. The complex morphology of the central regions of the galaxies is well captured by the models and resembles the visual classification given by Buta et al. (2015). A notable exception is the galaxy rings and spiral arms, which were not included in the fit, but masked out instead. The bumps in ellipticity shown in Figs 1 (at $\sim 9 \mathrm{kpc}$ ) and 2 (at $\sim 12 \mathrm{kpc}$ ) correspond to these non-fitted features. In addition, Buta et al. (2015) considered the presence of a barlens in NGC 5850 that we call the inner disc. The interpretation of this component in terms of the fitting function is ambiguous, as we use a Sérsic profile for fitting both barlenses and discs in the modelling. However, the MUSE TIMER velocity field clearly shows the presence of a fast-rotating component at the same spatial position as the inner disc (see Section 4). The case of the inner disc in NGC 1291 is less clear in the velocity field, probably because it is seen face-on (see discussion in Section 4).

\section{STELLAR KINEMATICS}

The 2D distributions of the stellar velocity, velocity dispersion, and higher-order Gauss-Hermite moments $h_{3}$ and $h_{4}$ (Gerhard 1993; van der Marel \& Franx 1993) have been computed for all the TIMER galaxies, as explained in Paper I and Gadotti et al. (2015). After Voronoi binning the data cubes to ensure a minimum signal-tonoise ratio $(\mathrm{SNR})>40$ per pixel (note that the original TIMER data cubes reach SNRs of 136 and 67 per pixel for NGC 1291 and NGC 5850, respectively), the pPXF code (Cappellari \& Emsellem 2004) was used in combination with the single stellar population (SSP) E-MILES models from Vazdekis et al. $(2012,2016)$ in order to fit the LOSVD for each spectrum corresponding to each bin. Potential emission lines were previously masked. More details on the binning procedure, the spectral range used for the fits, and the SSP models can be found in section 4.1 of Paper I.

Fig. 3 shows the four velocity moments for the two double-barred galaxies: NGC 1291 and NGC 5850. As expected, the presence of the inner bar is barely noticeable in the stellar velocity distribution, with only a minor distortion of the isovelocity contours observed in NGC 5850. NGC 1291 is a nearly face-on galaxy (inclination $i=$ $11^{\circ}$; see Section 2.1) and its inner bar is almost aligned with the line of nodes; in this case, no distortion of the isovelocity contours is expected (Du et al. 2016).

The most remarkable kinematic features are the $\sigma$-hollows, seen as blue regions at the ends of both inner bars in the corresponding subpanels of Fig. 3. The $\sigma$-hollows have amplitudes of $\Delta \sigma \sim$ $-40 \mathrm{~km} \mathrm{~s}^{-1}$ and $\sim-30 \mathrm{~km} \mathrm{~s}^{-1}$ for NGC 1291 and NGC 5850, respectively; these values are in full agreement with the results found in de Lorenzo-Cáceres et al. (2008) for NGC 5850 and the rest of their sample, for which the hollows showed amplitudes between -40 and $-10 \mathrm{~km} \mathrm{~s}^{-1}$

We note that $\sigma$-hollows have been observed in other doublebarred galaxies (de Lorenzo-Cáceres et al. 2012; de LorenzoCáceres et al. 2013; Du et al. 2016) and they seem, so far, an ubiquitous property of these systems. This result reinforces the validity of $\sigma$-hollows as kinematic diagnostics for identifying double-barred galaxies.

Apart from the inner bar, NGC 5850 hosts a kinematically decoupled inner disc as seen in the velocity map and confirmed by the expected $V-h_{3}$ anticorrelation (witnessed as more intense and opposite red and blue regions in the corresponding subpanels in Fig. 3; e.g. Bureau \& Athanassoula 2005). $h_{3}$ measures asymmetric deviations from the pure Gaussian behaviour of the LOSVD; a rotating disc generates a more extended LOSVD towards lowvelocity values and this is therefore detected with the $h_{3}$ moment. The kinematically observed inner disc in NGC 5850 corresponds 

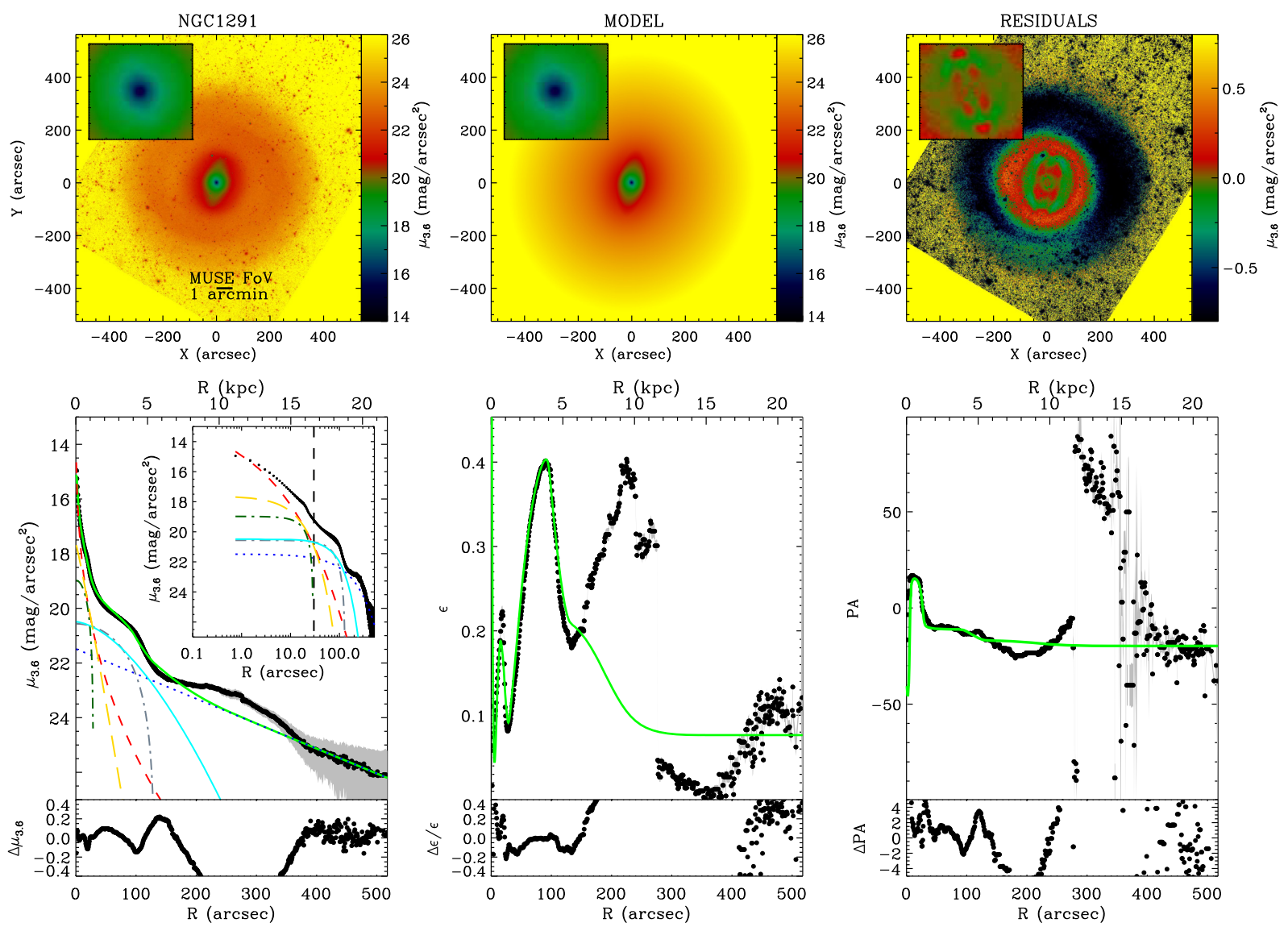

Figure 1. Two-dimensional multicomponent photometric decomposition performed with GASP2D for the double-barred galaxy NGC 1291. The top panels show the $\mathrm{S}^{4} \mathrm{G}$ image, the best-fitting 2D model, and the model-image residuals (from left to right). The insets zoom inside the corresponding MUSE FoV. The bottom left-hand panel shows the radial profile of the image with black dots and the same measurement for the model overplotted as a green line. This galaxy has been fitted with six structural components: bulge (red dashed line), inner bar (dark-green dash-dotted line), inner disc (yellow dashed line), outer bar (grey dash-dotted line), lens (cyan solid line), and galaxy disc (blue dotted line). The inset shows a zoom around the central regions, with the extent of the MUSE FoV indicated by a vertical dashed black line. The bottom middle and bottom right-hand panels show the ellipticity and position angle profiles for the $\mathrm{S}^{4} \mathrm{G}$ image (black dots) and the model (green line). All the bottom panels include a lower subpanel showing the corresponding residuals.

to the inner disc revealed by the photometric analysis described in Section 3; both analyses were performed independently and retrieved matching sizes for this inner component. The inner disc revealed by the photometric decomposition in NGC 1291 has no clear kinematic counterpart; this is not surprising as the almost faceon nature of this galaxy highly hampers the detection of rotational velocity features.

The higher-order Gauss-Hermite moment $h_{4}$ describes symmetric deviations of the LOSVD from a perfect Gaussian. The $h_{4}$ distribution for NGC 5850 shows a ring-shaped feature with positive values (white regions in the corresponding subpanel of Fig. 3). These kinds of rings have been found to be a kinematic property of inner bars seen almost face-on by means of numerical simulations (Du et al. 2016; Shen \& Debattista 2009). Fig. 10 in Du et al. (2016) shows that the presence of an inner-bar-associated $h_{4}$ ring is noticeable in galaxies with inclinations of $i \sim 30^{\circ}$, similar to that of NGC5850 $\left(i=39^{\circ}\right)$. Note that such an $h_{4}$ feature does not correspond to an actual ringed structure in the galaxy: It is a pure kinematic property that may indicate that inner bars are thinner than their surroundings. While the presence of the kinematically decoupled inner disc in NGC 5850 is already discussed in de Lorenzo-Cáceres et al. (2013), its positive- $h_{4}$ ring has only been unveiled thanks to the resolution of the TIMER data.
This demonstrates the necessity of both high spatial resolution and SNR to study the behaviour of these high-order moments that span short dynamical ranges.

NGC 1291 does not show the positive- $h_{4}$ ring but two absolute (within the MUSE FoV) maxima coincident with the position of the $\sigma$-hollows. These are seen as two white blobs in the $h_{4}$ distribution of NGC 1291 shown in Fig. 3. The $h_{4}$ distribution in NGC 1291, suggestive of the presence of a box/peanut structure, is the subject of a companion TIMER paper (Méndez-Abreu et al. 2019).

\section{STELLAR POPULATIONS}

\subsection{Full spectrum fitting with STECMAP}

The analysis of the stellar populations of the TIMER galaxies has been carried out with the STECMAP code (STEllar Content via Maximum A Posteriori; Ocvirk et al. 2006b), following the procedure extensively described in section 5.1 of Paper I and in Sánchez-Blázquez et al. (in preparation). Although we refer the reader to those papers for details on the use of STECMAP within TIMER, we repeat here the most relevant aspects of the process. First, we remark that the stellar-population analysis has been performed on the Voronoi-binned data described in Section 4 

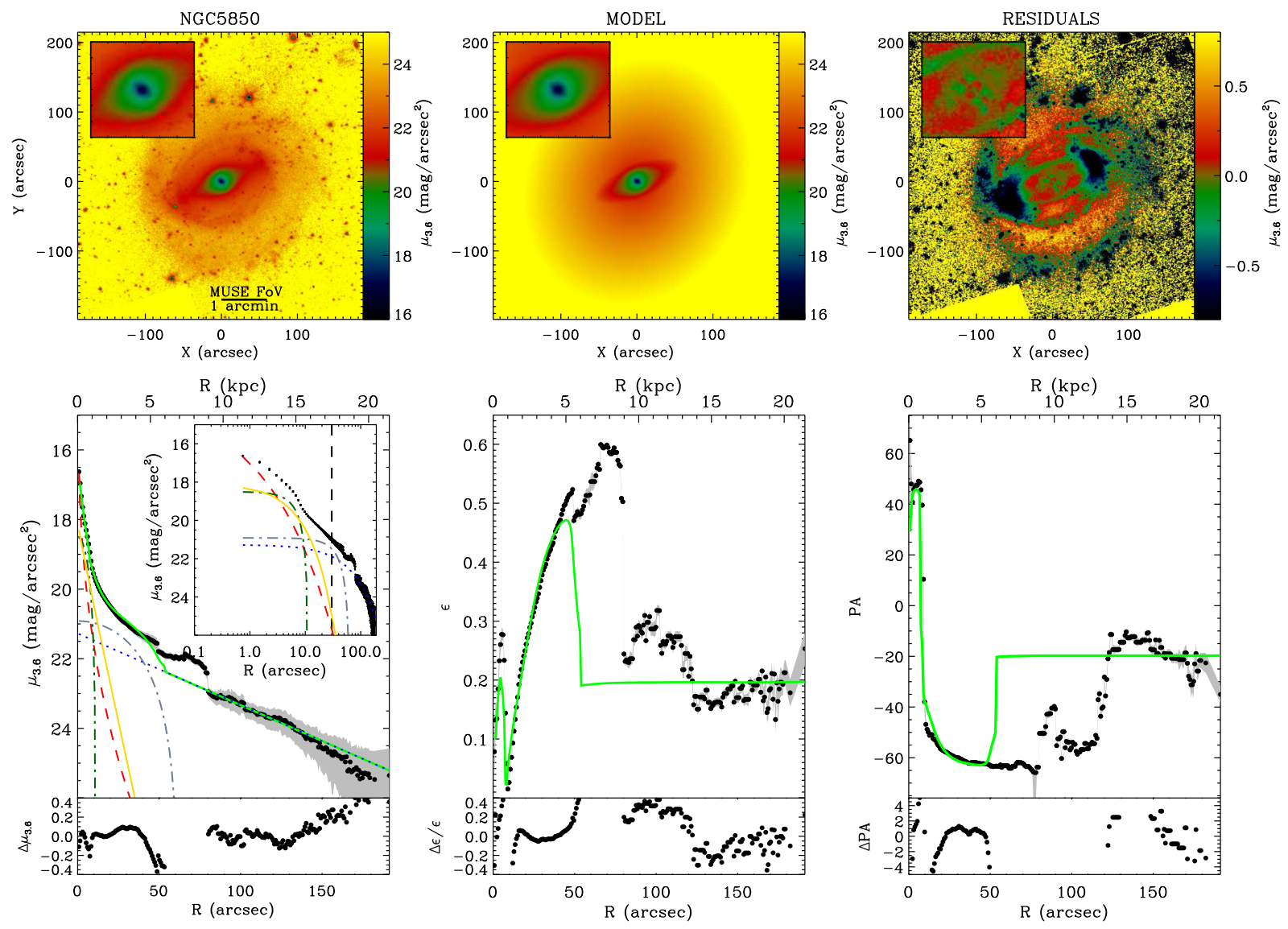

Figure 2. Same as Fig. 1 but for NGC 5850. This galaxy hosts five structural components: a bulge, inner bar, inner disc, outer bar, and galaxy disc. Unlike NGC 1291, there is no outer lens.

and that the gaseous contributions have been previously removed with GANDALF (Sarzi et al. 2006) in order to study pure stellar spectra.

STECMAP fits every spectrum corresponding to each bin with a linear combination of SSP models and a normalization curve. Note that, although fitting the kinematics simultaneously with the stellar populations is among the capabilities of STECMAP (in its version STECKMAP; Ocvirk et al. 2006a), we have kept the stellar kinematics fixed to the results obtained with PPXF. This is done to avoid degeneracies between the derived metallicity and the velocity dispersion (Koleva et al. 2007; Sánchez-Blázquez et al. 2011). As templates, we have again made use of the extended version of the MILES-based (Sánchez-Blázquez et al. 2006; Falcón-Barroso et al. 2011) models, E-MILES (Vazdekis et al. 2012, 2016), which cover the full spectral range of the TIMER data. In particular, models built with the BaSTI isochrones (Pietrinferni et al. 2013, and references therein) and following a Kroupa (2001) initial mass function are employed. Both data and templates are previously convolved to a constant spectral resolution of $2.8 \AA$, the maximum value of the wavelength-dependent MUSE resolution.

STECMAP allows the user to add a penalizing term to the minimizing function in order to regularize the solution. This penalization is used to constrain the best-fitting solution within physically meaningful limits, since retrieving the stellar populations with full-spectrum-fitting techniques is a highly degenerated and illconditioned problem. The choice of the most suitable regularization for the TIMER spectra is described in Paper I and Sánchez-Blázquez et al. (in preparation).

After running STECMAP on the TIMER data cubes, we obtain the relative contributions of populations of stars with different ages and metallicities within each Voronoi bin. Fig. 4 shows the 2D spatial distributions of the mean luminosity-weighted ages and metallicities for NGC 1291 and NGC 5850, computed by using the flux from the whole MUSE spectral range in each bin as weights. Both measurements have been averaged in linear scale. Note that, for the sake of enhancing all features, different age and metallicity ranges are shown for each galaxy in Fig. 4. Mean mass-weighted values have been obtained as well, providing analogous $2 \mathrm{D}$ distributions but with older values, as expected. Light-weighted measurements are used in the following as they enhance better the features of interest.

Results for NGC5850 are in good agreement with the mean luminosity-weighted ages and metallicities derived through measurement of line-strength indices in de Lorenzo-Cáceres et al. (2013): The inner bar appears younger ( $\sim 5 \mathrm{Gyr})$ and more metalrich than the surrounding regions corresponding to the outer bar (note that, due to the FoV, we did not probe beyond the outer bar in de Lorenzo-Cáceres et al. 2013). However, the higher spatial resolution provided by MUSE with respect to the SAURON integral-field spectrograph used in de Lorenzo-Cáceres et al. (2013) reveals more details on the complex age distribution inside and around the inner bar of NGC 5850. This will be further discussed in Section 6.2 . 
Table 1. Best-fitting parameters for the structural components of NGC 1291 and NGC 5850, obtained with the 2D photometric decompositions performed with GASP2D on the $3.6 \mu \mathrm{m}$ images.

\begin{tabular}{|c|c|c|c|}
\hline & & NGC 1291 & NGC 5850 \\
\hline \multirow[t]{6}{*}{ Bulge $^{a}$} & $\mu_{\mathrm{e}}\left(\operatorname{mag} \operatorname{arcsec}^{-2}\right)$ & 18.2 & 19.1 \\
\hline & $R_{\mathrm{e}}(\operatorname{arcsec})$ & 9.9 & 3.6 \\
\hline & $n$ & 2.97 & 2.68 \\
\hline & $b / a$ & 0.95 & 0.95 \\
\hline & $\mathrm{PA}\left({ }^{\circ}\right)$ & 29 & 55 \\
\hline & $\mathrm{B} / \mathrm{T}$ & 0.184 & 0.084 \\
\hline \multirow[t]{6}{*}{ Inner disc ${ }^{\mathrm{d}}$} & $\mu_{\mathrm{e}}\left(\operatorname{mag} \operatorname{arcsec}^{-2}\right)$ & 19.3 & 20.0 \\
\hline & $R_{\mathrm{e}}(\operatorname{arcsec})$ & 15.6 & 8.0 \\
\hline & $n$ & 0.92 & 1.04 \\
\hline & $b / a$ & 0.96 & 0.81 \\
\hline & $\mathrm{PA}\left({ }^{\circ}\right)$ & 155 & 147 \\
\hline & $D / T$ & 0.101 & 0.099 \\
\hline \multirow[t]{7}{*}{ Inner bar ${ }^{\mathrm{c}}$} & $\mu_{\mathrm{o}}\left(\mathrm{mag} \operatorname{arcsec}^{-2}\right)$ & 19.0 & 18.5 \\
\hline & $a(\operatorname{arcsec})$ & 29.0 & 11.3 \\
\hline & $n$ & 1.00 & 1.58 \\
\hline & $b / a$ & 0.35 & 0.21 \\
\hline & $\mathrm{PA}\left({ }^{\circ}\right)$ & 17 & 48 \\
\hline & $c$ & 2. & 2. \\
\hline & $\mathrm{Bar} / T$ & 0.018 & 0.017 \\
\hline \multirow[t]{7}{*}{ Outer bar ${ }^{c}$} & $\mu_{\mathrm{o}}\left(\mathrm{mag} \operatorname{arcsec}^{-1}\right)$ & 20.6 & 20.9 \\
\hline & $a(\operatorname{arcsec})$ & 131.5 & 62.1 \\
\hline & $n$ & 1.51 & 1.49 \\
\hline & $b / a$ & 0.43 & 0.41 \\
\hline & $\mathrm{PA}\left({ }^{\circ}\right)$ & 170 & 115 \\
\hline & $c$ & 1.9 & 1.8 \\
\hline & $\mathrm{Bar} / T$ & 0.087 & 0.109 \\
\hline \multirow[t]{6}{*}{ Lens $^{\mathrm{d}}$} & $\mu_{\mathrm{e}}\left(\operatorname{mag} \operatorname{arcsec}^{-2}\right)$ & 121.5 & N/A \\
\hline & $R_{\mathrm{e}}(\operatorname{arcsec})$ & 75.3 & N/A \\
\hline & $n$ & 0.61 & N/A \\
\hline & $b / a$ & 0.73 & N/A \\
\hline & $\mathrm{PA}\left({ }^{\circ}\right)$ & 163 & N/A \\
\hline & $L / T$ & 0.206 & N/A \\
\hline \multirow[t]{5}{*}{ Disc $^{b}$} & $\mu_{\mathrm{o}}\left(\mathrm{mag} \operatorname{arcsec}^{-2}\right)$ & 21.5 & 21.3 \\
\hline & $h(\operatorname{arcsec})$ & 119.1 & 52.7 \\
\hline & $b / a$ & 0.92 & 0.80 \\
\hline & $\mathrm{PA}\left({ }^{\circ}\right)$ & 160 & 160 \\
\hline & $D / T$ & 0.40 & 0.692 \\
\hline
\end{tabular}

${ }^{a}$ The bulges are parametrized with a Sérsic function (Sérsic 1968) where $R_{\mathrm{e}}, \mu_{\mathrm{e}}, n, b / a, \mathrm{PA}$, and $B / T$ are the effective (or half-light) radius, the surface brightness at $R_{\mathrm{e}}$, the Sérsic index describing the curvature of the profile, the axial ratio, the position angle, and the contribution of the bulge to the total galaxy light, respectively.

${ }^{b}$ The galaxy discs are described with a single exponential profile where $\mu_{0}$, $h, b / a, \mathrm{PA}$, and $D / T$ are the central surface brightness, scale length, axial ratio, position angle, and contribution of the disc to the total galaxy light, respectively.

${ }^{c}$ The bars are described with a Ferrers profile (Ferrers 1877), where $\mu_{0}, a$, $b / a, \mathrm{PA}$, and Bar/T represent the central surface brightness, length, axial ratio, and contribution of the bar to the total galaxy light, respectively. The shape parameters are $n$, which describes the shape of the surface brightness profile, and $c$, which controls the shape of the isophotes following generalized ellipses (boxy when $c>2$ versus discy when $c<2$; Athanassoula et al. 1990).

${ }^{d}$ The inner discs and lens are parametrized with Sérsic profiles analogous to those describing the bulges.

Unlike all double-barred galaxies for which analysis of stellar populations is available to date, which show that inner bars are younger and more metal-rich than outer bars (de Lorenzo-Cáceres et al. 2012, 2013), NGC 1291 hosts an inner bar that is older than the outer bar. Even though the dynamical range for the ages in this galaxy is very limited (the colour bar in Fig. 4 covers from 5 to 8 Gyr, with a characteristic error bar of $\sim 0.9 \mathrm{Gyr}$ ), the mean luminosityweighted age map clearly traces the inner-bar shape with older ages than the surroundings. Such an effect is hardly due to errors in the measurements alone. This surprising result will be discussed in Section 6.2.

The metallicity map for NGC 1291 does show the higher values for the inner bar found for other double-barred cases (de LorenzoCáceres et al. 2013; although, once again, note the short dynamical range of this parameter). For both galaxies, the very centres within the inner bars, where the metallicity reaches the highest values, show old ages up to 9 Gyr.

\subsection{Dissecting the galaxies: segmentation maps}

Significant information is contained in the best-fitting solutions provided by STECMAP for the stellar population content of the TIMER galaxies. Azimuthal profiles are not a good approach as the structural complexity of the central regions of double-barred galaxies makes the isophotal ellipses round due to the overlapping of many components. As a result, the azimuthally averaged profile will mix information from various structures. This is well illustrated with the case of the inner bar of NGC 1291, for which the isodensity contours plotted over the maps throughout this paper (white lines) are much rounder than the inner bar alone (black lines).

Since we aim at discussing the structural assembly history of double-barred systems with the SFH results from STECMAP, our strategy consists of comparing the measurements within inner bars with those within other components, namely bulges, outer bars, inner discs, lenses, and discs. For this purpose, we average the relative contributions of the different stellar populations over apertures where the light contribution of each structure is mostly dominant. This approach is particularly important in structurally complex galaxies such as those presented here.

Fig. 5 shows the relative light contribution from each structural component to every MUSE spaxel for the two galaxies under study. These maps are built by taking the analytical description of each structure (obtained from the best fit of the 2D photometric decompositions described in Section 3) and projecting it on to a MUSE FoV with the MUSE spatial resolution. The total addition of these maps (whose minimum and maximum values are indicated in Tables 2 and 3) generates a flat image with a value of 1 .

We must note here that the effect of the MUSE PSF is not taken into account in the structure maps shown in Fig. 5: The PSF affects the total galaxy image, but it has no physical meaning to apply it to each 2D structure model separately. Dismissing the PSF has the highest impact on the recovery of the light contribution of the very central structures (mainly bulge) and, for this reason, very central regions are considered separately in the following analysis. The characteristic PSF FWHM for each galaxy is computed as the median value for all the individual exposures involved in the final TIMER data cube and it acquires a value of $1.1 \operatorname{arcsec}$ and 1 arcsec for NGC 1291 and NGC 5850, respectively.

The information provided by Fig. 5 is then used to design segmentation maps defining which regions of the MUSE FoV are dominated (or almost dominated) by each structural component. The Voronoi binning applied to the data is considered at this stage, as results within bins and not within spaxels are going to be averaged. The final segmentation maps for NGC 1291 and NGC 5850 shown in Figs 6 and 7, respectively, are built by considering a central circular region accounting for PSF effects and defined with a radius 

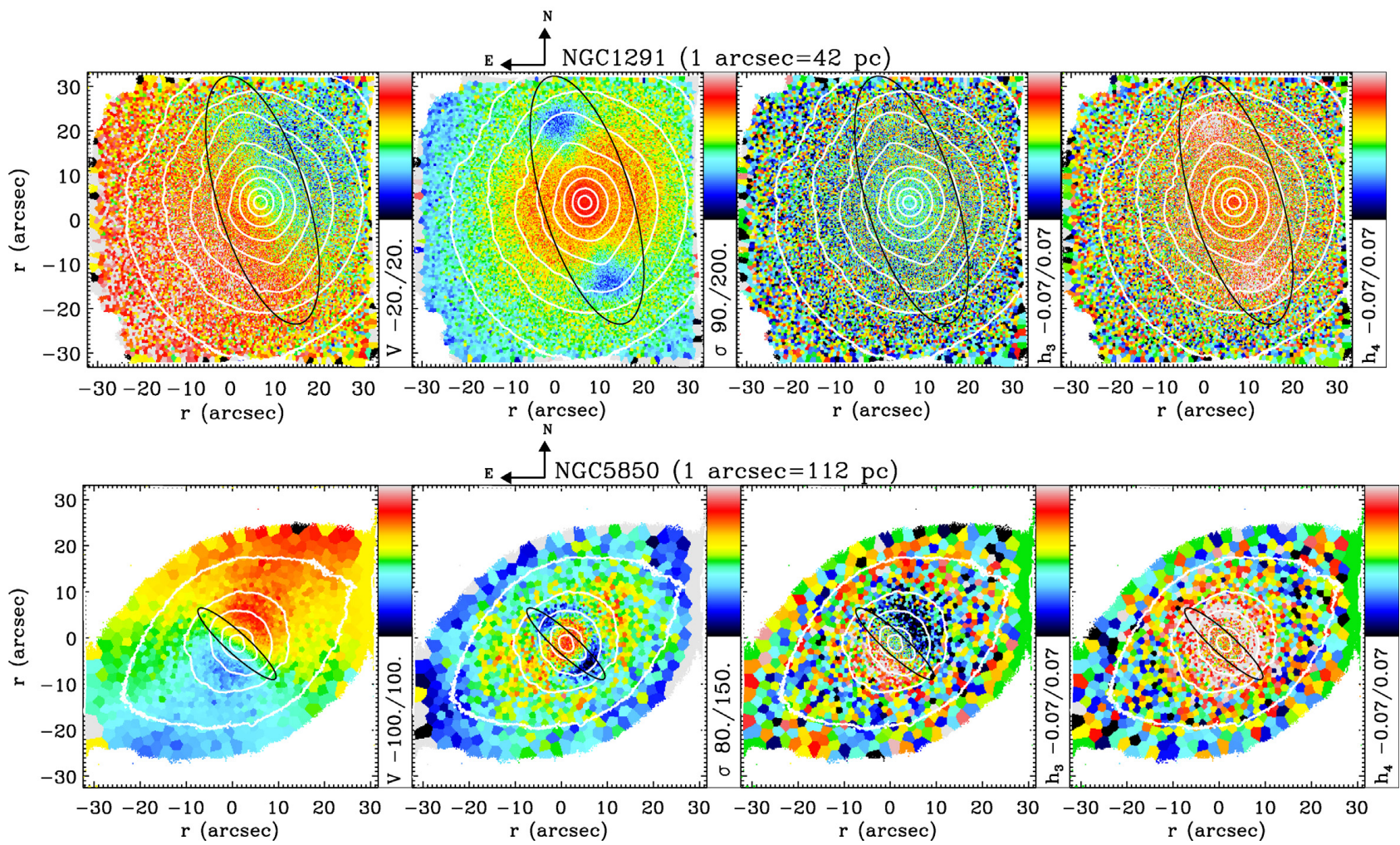

Figure 3. Two-dimensional distribution of the line-of-sight stellar kinematic moments for NGC 1291 (top panels) and NGC 5850 (bottom panels). For each galaxy and from left to right, the subpanels correspond to the stellar velocity $V$ in $\mathrm{km} \mathrm{s}^{-1}$, velocity dispersion $\sigma$ in $\mathrm{km} \mathrm{s}^{-1}, h_{3}$, and $h_{4}$. Median errors for the four parameters are $\left(5 \mathrm{~km} \mathrm{~s}^{-1}, 6 \mathrm{~km} \mathrm{~s}^{-1}, 0.03,0.03\right)$ for NGC 1291 and $\left(4 \mathrm{~km} \mathrm{~s}^{-1}, 6 \mathrm{~km} \mathrm{~s}^{-1}, 0.03,0.04\right)$ for NGC 5850. Flux contours are overplotted as solid white lines; the inner bar (full length of the Ferrers profile) is outlined in black using the results from the 2D photometric decomposition. The presence of the inner bars is clearly noticeable in the velocity dispersion maps, where two $\sigma$-hollows appear at both ends of the inner bars. The inner disc in NGC 5850 is revealed by the velocity and $h_{3}$ distributions.

equivalent to $3 \sigma$ of the PSF; besides, the regions of dominance for each structural component are built with the selection criteria detailed in Tables 2 and 3. Some remarks about this selection:

(i) All segments are intentionally chosen to be non-adjacent. In some cases, this is achieved by imposing tighter constraints on the light contribution from some structures (see e.g. the criterion on the bulge light when selecting the segment corresponding to the inner disc in NGC 5850).

(ii) Two bulge regions are considered for NGC 1291: an outer bulge, which is not contaminated by the inner bar (inner bar light contribution $<0.5$ percent), and an inner bulge, where the light contribution from the inner bar, while lower than that from the bulge, is not negligible. Given the closeness of NGC 1291, the central regions are mapped with superb spatial resolution and the selection of an inner-bulge segment allows us to analyse gradients with respect to the outer-bulge and inner-bar regions.

(iii) The spatial resolution of NGC 5850 allows us to select one bulge segment only. As in the outer bulge of NGC 1291, an almostzero contribution from the inner bar $(<0.5$ per cent $)$ is required in order to avoid any overlapping.

(iv) The bulge in NGC 5850 dominates the light over the inner disc only in the very central regions, where the circular region accounting for PSF effects is located. For that reason, the criterion for the inner disc when selecting the bulge segment has been relaxed with respect to the other structures. (v) The brightest regions for the inner bar of NGC 1291 have a similar surface brightness as the bulge and inner disc in the same bins. This also happens for the outer bar with respect to the lens.

(vi) The galaxy disc in NGC 1291 never dominates the light within the MUSE FoV and, therefore, no disc segment is defined. Note however that the underlying disc is to some extent affecting the measurements for all the structures.

The purpose of avoiding defining adjacent segments is twofold: First, since the information of the light contribution from each structure comes from the 2D photometric decompositions performed on the $\mathrm{S}^{4} \mathrm{G}$ images, it does not necessarily match the light distribution in the optical range where MUSE works. For this reason we select those regions where the contribution from each structure is the highest, which will be most likely similar for all bandpasses. Second, by making the largest differentiation between structures we minimize contamination as much as possible, although we remark that each aperture actually accounts for several overlapping structures except for, most probably, the region dominated by the galaxy disc in the case of NGC 5850.

Once the segmentation maps are created, the results from the STECMAP analysis described in Section 5 are averaged within every structure and compared, as described in the following Sections 5.3 and 5.4 . 

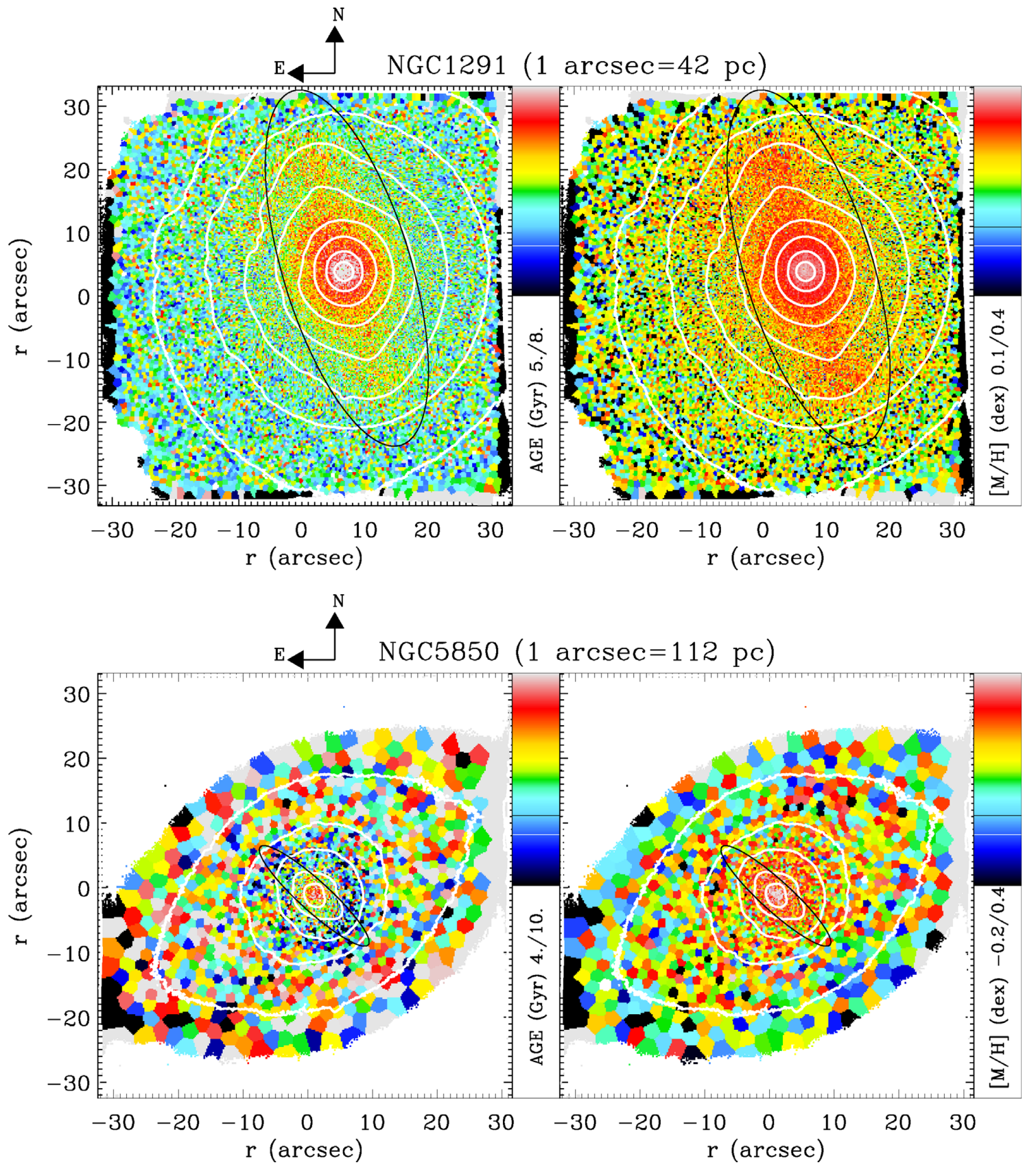

Figure 4. 2D distributions of the mean luminosity-weighted ages (left-hand subpanels) and metallicities (right-hand subpanels) for NGC 1291 (on top) and NGC 5850 (on bottom). Median error bars are of 0.9 Gyr and 0.04 dex for NGC 1291 and of 0.7 Gyr and 0.06 dex for NGC 5850. Flux contours are overplotted as solid white lines and the inner bar (full length of the Ferrers profile) is outlined in black by using the results from the 2D photometric decomposition. Different age and metallicity ranges are used for each galaxy to enhance the various features. The inner bar in NGC 1291 appears older and more metal-rich than the surroundings, the inner bar in NGC 5850 being younger and more metal-rich.

\subsection{The star formation history of NGC 1291 at a glance}

Fig. 6 shows the fraction of light contributed by stars of different ages, as well as the metallicities, for the different structural components. The behaviour of the stellar age distribution is similar for all structures: Between 40 percent and 50 percent of the light comes from stars that were formed more than $7 \mathrm{Gyr}$ ago (dismissing the central region accounting for PSF effects, for which the fraction of light from old stars increases up to $\sim 60$ per cent). The star formation process smoothly decayed until $\sim 3 \mathrm{Gyr}$ ago, when it was rapidly quenched such that stars younger than $\sim 1$ Gyr contribute less than 1 per cent to the total galaxy light.

While the stellar age distributions for the outermost components, particularly the lens and outer bar, are exactly the same, a gradient among the innermost structures is noticeable. The centre presents the highest contribution from old stars, which progressively decays for the inner bulge, inner bar, and outer bulge. These results explain the behaviour observed in the mean luminosity-weighted ages and metallicities shown in Fig. 4, where the inner bar appears as a 

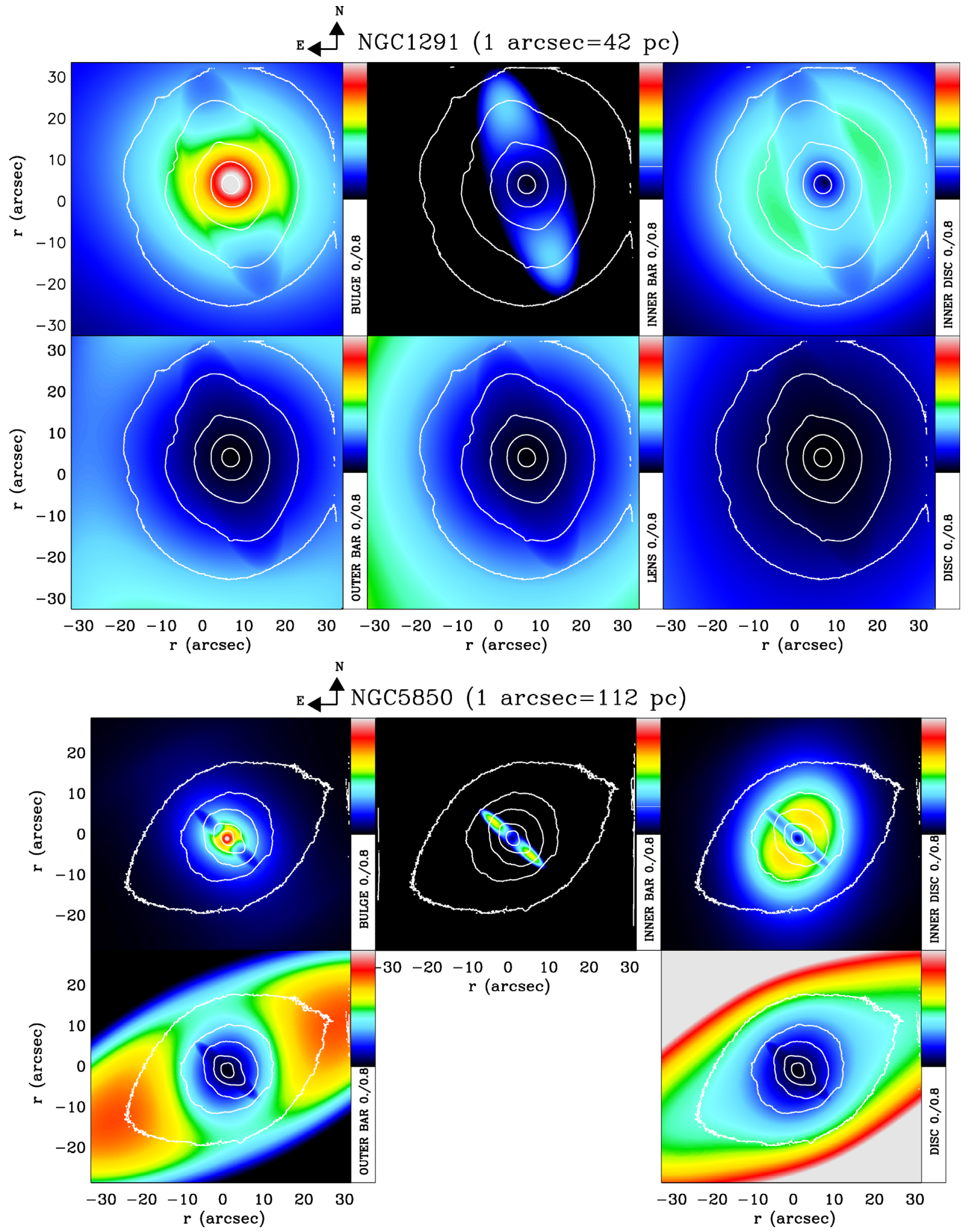

Figure 5. Spatial distribution of the relative light contribution from each structural component to the total surface brightness of each galaxy (NGC 1291 in the top panels; NGC 5850 in the bottom panels) within each spaxel, as modelled with the 2D photometric decompositions described in Section 3: bulge (top left-hand subpanel), inner bar (top middle subpanel), inner disc (top right-hand subpanel), outer bar (bottom left-hand subpanel), lens (bottom middle subpanel, when applicable), and galaxy disc (bottom right-hand subpanel). For the sake of clarity, all subpanels show values ranging from 0 per cent to 80 per cent of light contribution from each structure to the total galaxy light at each spaxel. The addition of all panels would provide a flat image of value 1 (i.e. 100 per cent). The images match the FoV and spatial resolution of the TIMER data for these galaxies. The Voronoi binning is not applied at this stage. Flux contours for the actual MUSE data are overplotted as white solid lines. 
Table 2. Selection of the MUSE bins whose light is dominated by every structural component in NGC 1291. Each row corresponds to one individual component. The first two columns indicate the minimum and maximum relative contribution from that component to the total galaxy light within the FoV. The remaining columns indicate the criteria of light contribution from all the components used for creating the segmentation map shown in Fig. 6 (top left-hand panel). Two bulge-dominated regions are considered for this galaxy (see text for details). The disc never dominates within the MUSE FoV.

\begin{tabular}{|c|c|c|c|c|c|c|c|c|}
\hline Structure & Min \% light & Max \% light & Bulge & Inner bar & Inner disc & Outer bar & Lens & Disc \\
\hline INNER BULGE & $7 \%$ & $97 \%$ & $\geq 60 \%$ and $\leq 75$ & $<60 \%$ & $<60 \%$ & $<60 \%$ & $<60 \%$ & $<60 \%$ \\
\hline OUTER BULGE & $7 \%$ & $97 \%$ & $\geq 45 \%$ & $<0.5 \%$ & $<45 \%$ & $<45 \%$ & $<45 \%$ & $<45 \%$ \\
\hline INNER BAR & $0 \%$ & $27 \%$ & $<28 \%$ & $\geq 25 \%$ & $<28 \%$ & $<25 \%$ & $<25 \%$ & $<25 \%$ \\
\hline INNER DISC & $2 \%$ & $38 \%$ & $<35 \%$ & $<35 \%$ & $\geq 35 \%$ & $<35 \%$ & $<35 \%$ & $<35 \%$ \\
\hline OUTER BAR & $0 \%$ & $35 \%$ & $<25 \%$ & $<25 \%$ & $<25 \%$ & $\geq 25 \%$ & $<27 \%$ & $<25 \%$ \\
\hline LENS & $0 \%$ & $51 \%$ & $<30 \%$ & $<30 \%$ & $<30 \%$ & $<30 \%$ & $\geq 30 \%$ & $<30 \%$ \\
\hline DISC & $0 \%$ & $24 \%$ & - & - & - & - & - & - \\
\hline
\end{tabular}

Table 3. Same as Table 2 but for NGC 5850. The corresponding segmentation map is shown in Fig. 7 (top left-hand panel).

\begin{tabular}{lccccccc}
\hline Structure & Min $\%$ light & Max \% light & Bulge & Inner bar & Inner disc & Outer bar & Disc \\
\hline BULGE & $0 \%$ & $91 \%$ & $\mathbf{2 3 5 \%}$ & $<0.5 \%$ & $<50 \%$ & $<35 \%$ & $<35 \%$ \\
INNER BAR & $0 \%$ & $48 \%$ & $<40 \%$ & $\geq \mathbf{4 0 \%}$ & $<40 \%$ & $<40 \%$ & $<40 \%$ \\
INNER DISC & $0 \%$ & $52 \%$ & $<20 \%$ & $<40 \%$ & $\geq \mathbf{4 0 \%}$ & $<40 \%$ & $<40 \%$ \\
OUTER BAR & $0 \%$ & $63 \%$ & $<40 \%$ & $<40 \%$ & $<10 \%$ & $\geq \mathbf{5 0 \%}$ & $<40 \%$ \\
DISC & $0 \%$ & $99 \%$ & $<40 \%$ & $<40 \%$ & $<40 \%$ & $<40 \%$ & $\geq \mathbf{5 0 \%}$ \\
\hline
\end{tabular}

slightly older component than the surrounding structures (inner disc, outer bar, and lens).

Differences among the inner structures (centre, inner and outer bulge, and inner bar) are most apparent in the two top-right subpanels of Fig. 6, where the spatial distributions of the total light contributions from stars older than $7 \mathrm{Gyr}$ and stars between 1 and 2 Gyr old are shown. This latest age range enhances small differences $(<10$ percent) in the contribution from young stars, as the centre and inner bulge appear in blue colours, the inner bar looks greener, and the outer regions are all coloured in yellow/red.

All structural components show similar metallicity values with a subtle gradient which will be discussed in Section 6.2, where these results will be used to constrain the dynamical assembly process of NGC 1291.

\subsection{The star formation history of NGC 5850 at a glance}

Fig. 7 shows the corresponding stellar-population analysis for NGC 5850. Significant differences among the inner bar, the rest of the inner structures (bulge and inner disc), and the outermost components (outer bar and galaxy disc) are found in this case.

The most distinct stellar age distribution is that of the inner bar, which shows a bump indicating a higher light fraction ( $\sim 50$ per cent) coming from stars with ages between 1 and $4.5 \mathrm{Gyr}$. This feature is used to show the different contributions from young stars in the inner bar region in the top right-hand subpanels of Fig. 7; stars older than 7 Gyr are also shown, as done for NGC 1291.

The bulge shows a smoothly declining profile going from light fractions of $\sim 16$ per cent for very old ages to 6 percent for stars of $\sim 3 \mathrm{Gyr}$, when a sudden change of slope occurs and the profile abruptly decays. Both the inner bar and the bulge are more metalrich than the outermost components (disc and outer bar).

We reiterate here that the inner disc has been detected from both the photometric decomposition and kinematic analyses in an independent manner. Moreover, it is dominant in the bulge region. Indeed both components show a similar stellar age distribution but with a smaller contribution from intermediate-age populations (between 4 and $10 \mathrm{Gyr}$ ) in the inner disc, which is also less metalrich than the bulge.

Twenty per cent of the outer bar light comes from very old stars and the light fraction decreases until it reaches $<1$ percent due to stars younger than $1.3 \mathrm{Gyr}$. The contribution from stars younger than $1 \mathrm{Gyr}$ is indeed negligible for all the structural components, as in NGC 1291. The stellar age distribution for the disc is very similar to that of the outer bar, with a subtle rejuvenation noticeable when comparing ages older and younger than $\sim 6$ Gyr. As seen in Fig. 4, the centre stands out with older and more metal-rich populations. A gradient in metallicities is observed, from metal-rich to less metalrich inside outwards.

\subsection{Measurement of the $[\alpha / \mathrm{Fe}]$ overabundance distribution}

In order to retrieve more information about the time-scales governing the formation process for the double-barred galaxies, we derived the $2 \mathrm{D}$ spatial distribution of the $[\alpha / \mathrm{Fe}]$ overabundance. This was done by using a Bayesian fitting of the measurements of the line-strength indices with the predictions for the same indices measured over the solar-scaled and $\alpha$-enhanced MILES-based SSP models of Vazdekis et al. (2015). The line-strength indices used in the fit were $\mathrm{H} \beta_{\mathrm{o}}$ (Cervantes \& Vazdekis 2009), Fe4383 and Fe5015 (Worthey et al. 1994), and Fe5270 and Mgb (Burstein et al. 1984). They were selected for a best description of the age, metallicity, and $[\alpha / \mathrm{Fe}]$ within the MUSE spectral range. This statistical technique has already been successfully used in Martín-Navarro et al. (2018) to retrieve $[\alpha / \mathrm{Fe}]$ values, as well as single ages and metallicities. We refer the reader to that paper for details on how the index-fitting works and the selection of the indices.

Fig. 8 shows the $[\alpha / \mathrm{Fe}] 2 \mathrm{D}$ distributions for NGC 1291 and NGC 5850. Inner bars appear with bluer colours than the rest of the FoV, meaning they have lower $[\alpha / \mathrm{Fe}]$ values than the outermost structures. This result suggests inner bars have undergone a longer formation process than the inner disc, outer bar, and lens or galaxy 


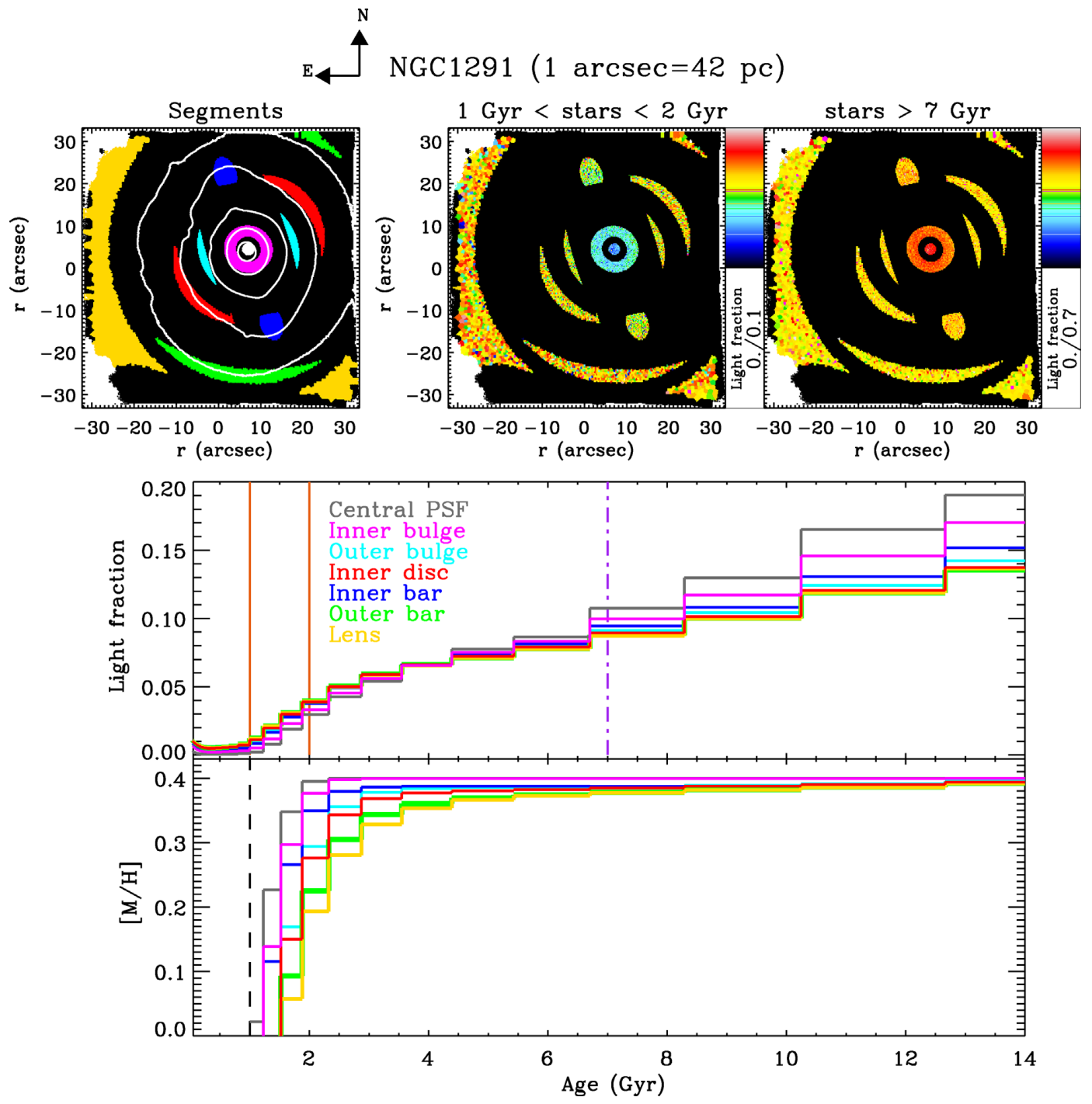

Figure 6. Analysis of the SFH for the double-barred galaxy NGC 1291. The top left-hand panel is the segmentation map defining the regions where each structural component dominates the total galaxy light (see text for details). Flux contours for the actual MUSE data are overplotted as white solid lines. The top right-hand panels show the 2D distribution of stellar populations within a range of young ages (left-hand panel) and for ages older than a threshold (right-hand panel), within the structures of interest. The profiles in the bottom panels correspond to the contribution from stellar populations of different ages to the total galaxy light (top), and the age-metallicity relation (bottom) within each structural component. Although light fractions and metallicities are computed for each discrete age, a continuous histogram-like profile is shown instead for the sake of clarity. Note there is a significant overlap between the light fractions for the outer bar and lens. The vertical solid orange and dash-dotted purple lines indicate the age regions used for computing the top averaged maps. Stellar populations younger than $\sim 1$ Gyr contribute less than 1 per cent and they are not shown in the age-metallicity profile.

disc, since the time-scales involved in the creation of $\alpha$ elements are shorter than those responsible for Fe formation.

\section{DISCUSSION}

\subsection{Nature versus nurture of the $\sigma$-hollows}

The $\sigma$-hollows observed in all double-barred galaxies studied to date may provide information about the dynamics of inner bars, but their origin is still unclear. de Lorenzo-Cáceres et al. (2008) suggested that $\sigma$-hollows are not intrinsic features of the velocity dispersion distribution of any isolated structure (inner bar, disc, or bulge). Within their explanation, inner bars have a rather flat and cold profile of the velocity dispersion along them, while hot classical bulges show a decaying distribution from high values and discs have a smooth axisymmetric distribution with still higher $\sigma$ values than the inner bar at radii matching with the inner bar size. The coexistence of these three structures produces a visual effect such as local minima due to the low- $\sigma$ values of the inner bar being observed only where the inner bar dominates the total galaxy light over the hotter bulge and hotter (at those radii) disc, i.e. at the bar ends. If this hypothesis (which we refer to as nurture of the $\sigma$-hollows) was correct, the appearance of the $\sigma$-hollows would depend on the relative contribution of the structures involved (mainly inner bar and bulge in the previous example) to the total light, as well as on their relative level of rotational versus pressure support. Indeed $\sigma$-hollows would not be seen in galaxies without a bulge (or other central structure with a different dispersion value with respect to the inner bar). 


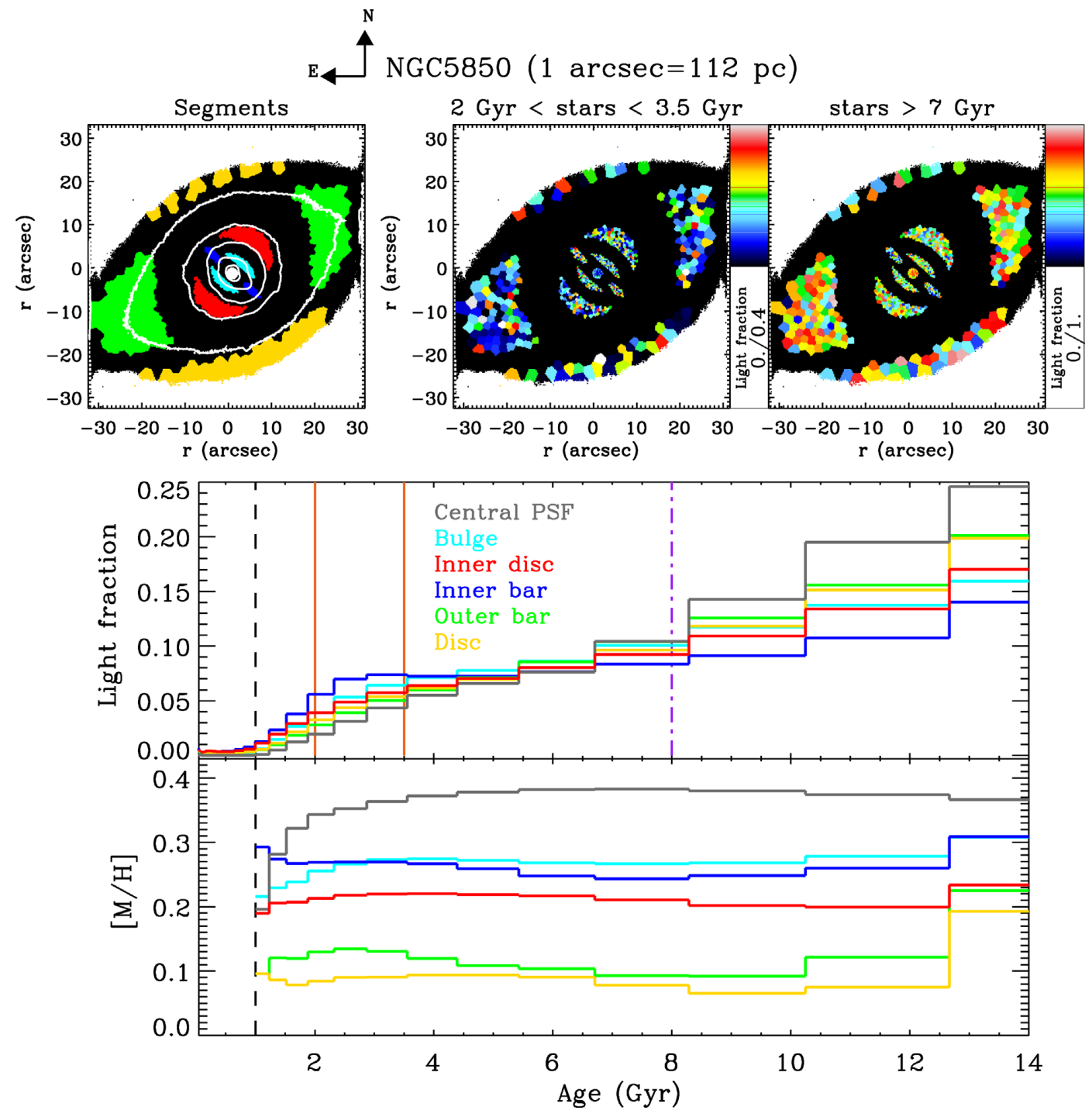

Figure 7. Same as Fig. 6 but for the double-barred galaxy NGC 5850.

More recently, Du et al. (2017) used numerical simulations to show that the presence of any bar, either single or embedded in a double system, produces features in the orthogonal velocity dispersion distribution of its host disc. Indeed, the pure $\sigma_{z}$ of the disc (once isolated from all other structures, including the bar) shows local minima at the location of the bar ends and local maxima in the perpendicular direction. When analysing the total velocity dispersion distribution of a projected system composed of a bar and disc, these features resemble the observed $\sigma$-hollows, as well as two perpendicular bumps termed as $\sigma$-humps. Simulations also show that the smaller the bar, the more intense both $\sigma$-hollows and humps appear. Why the $\sigma_{z}$ of the disc is modified by the presence of a bar and why smaller bars produce more enhanced $\sigma$ features remain unclear.

In this case the presence of a bulge or any other central hotter/cooler structure than the inner bar is not needed. Indeed, a hot bulge may mask the detection of these $\sigma$ features, although the $\sigma$-hollows, more prominent than the humps, usually remain. We reiterate that the disc hosting the bar is responsible for the $\sigma$ hollows, which are physically produced in it: Its intrinsic velocity dispersion does decay at those points.
Whether the $\sigma$-hollows are a matter of visual contrast among structures or have a physical nature in the galaxy disc is unknown. In an attempt to shed some light on this topic, Fig. 9 shows the 2D velocity dispersion distribution for NGC 1291 and NGC 5850 together with the profiles extracted from pseudo-slits along the direction of each inner bar and perpendicular to them. The $\sigma$ hollows are clearly observed in the profiles along the inner bars. The strong inner bar in NGC 5850 is rather narrow in the perpendicular direction; it is therefore complicated to extract conclusions about possible local maxima for this galaxy, as predicted by the scenario of $\mathrm{Du}$ et al. (2017). NGC 1291 does show features along the perpendicular direction but at smaller radii, and they are better interpreted as local minima rather than local maxima.

The sizes of the bulges and inner bars for both galaxies have been overplotted in Fig. 9. The corresponding ellipses are drawn by considering the effective radii of the bulges (in yellow), the effective radii of the inner bars (in blue), and the full length of the Ferrers profiles for the inner bars (in black). Moreover, the radii at which the surface brightness from bulge and inner bars are the same are indicated with circles (magenta for the innermost crossing, brown for the outermost crossing). This means that the bins inside the 

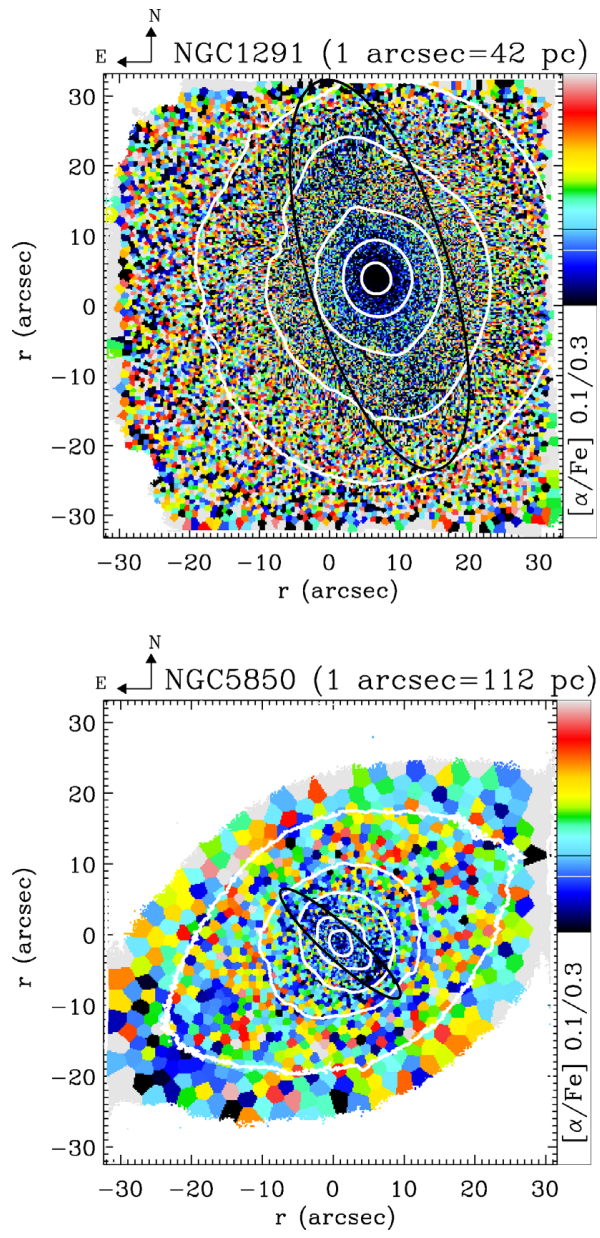

Figure 8. 2D spatial distribution of the $[\alpha / \mathrm{Fe}]$ overabundance for NGC 1291 (top) and NGC 5850 (bottom). Typical error bars are of $\sim 0.03$ dex for NGC 1291 and $\sim 0.11$ dex for NGC 5850. Flux contours are overplotted as solid white lines. The inner bar (full length of the Ferrers profile) is outlined in black by using the results from the 2D photometric decomposition.

inner bar and delimited by the magenta and brown circles are those where the surface brightness of the inner bar is greater than that of the bulge.

It is noticeable that the $\sigma$-hollows appear at the region where the light coming from the individual inner bar dominates over the bulge light, even for the case of NGC 1291, where the levels of brightness from both structures are very close, as seen in the inset of Fig. 1. This result supports the nurture origin of the $\sigma$-hollows, which are indeed visible where the inner bar dominates over the presumably hotter bulge and other cooler structures (e.g. inner disc).

We also emphasize that both galaxies host bulges with relatively high Sérsic indices $(n=2.97$ and 2.68 for NGC 1291 and NGC 5850, respectively), pointing towards a classical origin for them. Classical bulges are expected to be dynamically hot structures. Moreover, in Section 6.3 we will introduce the finding of a box/peanut structure at the centre of NGC 1291, as found by Méndez-Abreu et al. (2019); box/peanuts are thicker than their host bars and therefore they have higher velocity dispersion values as well.

Finally, we warn the reader that although these pieces of evidence support the scenario proposed in de Lorenzo-Cáceres et al. (2008), a physical origin for the $\sigma$-hollows cannot be fully discarded as there is a chance these regions fortuitously coincide with the alterations of the disc $\sigma_{z}$ obtained by Du et al. (2017).

\subsection{How double-barred galaxies form}

Our stellar population results for the individual structural components in the two double-barred galaxies shed light on the formation paths NGC 1291 and NGC 5850 have followed. The first milestone in this discussion is the finding of an inner-disc component for both galaxies, whose size matches those of the inner bars. While the inner disc in NGC 5850 had been already detected by kinematic analysis (e.g. de Lorenzo-Cáceres et al. 2013), the face-on condition of NGC 1291 prevents the kinematic identification of the inner disc, which has been detected with the $2 \mathrm{D}$ photometric decompositions instead.

Within the two possible formation scenarios described in Section 1, scenario 1 invokes a direct formation of the inner bar after gas inflow through the outer bar. This represents a special way of forming bars, as normal bars (i.e. single bars or large-scale bars within double-barred systems) are formed through dynamical instabilities from cold discs (e.g. Combes et al. 1990; Debattista \& Sellwood 2000; Athanassoula 2003). Inner and outer bars may therefore be considered intrinsically different within this scenario. It is worth noting that inner bars do not usually present gaseous counterparts (Petitpas \& Wilson 2004; but see the case of NGC 2273 in Petitpas \& Wilson 2002).

On the other hand, in scenario 2 both inner and outer (or single) bars follow the same formation process; i.e. both are born from instabilities in dynamically cold discs. While large-scale bars are formed in galaxy discs, inner bars are embedded in inner discs. Inner discs are a rather frequent structural component, appearing in $\sim 29$ per cent of ellipticals and S0s (Krajnović et al. 2008; estimate from kinematic analyses). This scenario therefore considers that all bars share the same nature and they may therefore undergo similar evolutionary processes, which may be different only when differences in size play a role.

The discovery of inner discs in our two galaxies supports this second scenario, further backed by the fact that the inner bar in NGC 1291 appears older than the outer bar. This possibility is not accounted for in scenario 1, where the outer bar needs to be formed prior to the inner bar, while scenario 2 does not impose any sequential requirement. The fact that the inner discs may be very faint, as in the case of NGC 1291, would explain why they have not been detected in all double-barred galaxies observed. We remind the reader here that most of the studies available for double-barred galaxies are photometrically based; however, the spectroscopic analyses of de Lorenzo-Cáceres et al. (2012) and de Lorenzo-Cáceres et al. (2013) do find kinematic signatures of inner discs in four out of five double-barred galaxies under study. The remaining galaxy hosts a very small inner bar and resolution effects may have prevented the detection of the inner disc in this case.

The measurements shown in Section 5 indicate that NGC 1291 and NGC 5850 have undergone formation processes with different pathways and therefore they are discussed separately, as follows.

\subsubsection{NGC 1291: a rather contemporary assembly of all structures at an early epoch}

NGC 1291 shows a similar SFH for all the structures in place within the MUSE FoV; from inside outwards: centre, bulge, inner bar, inner 

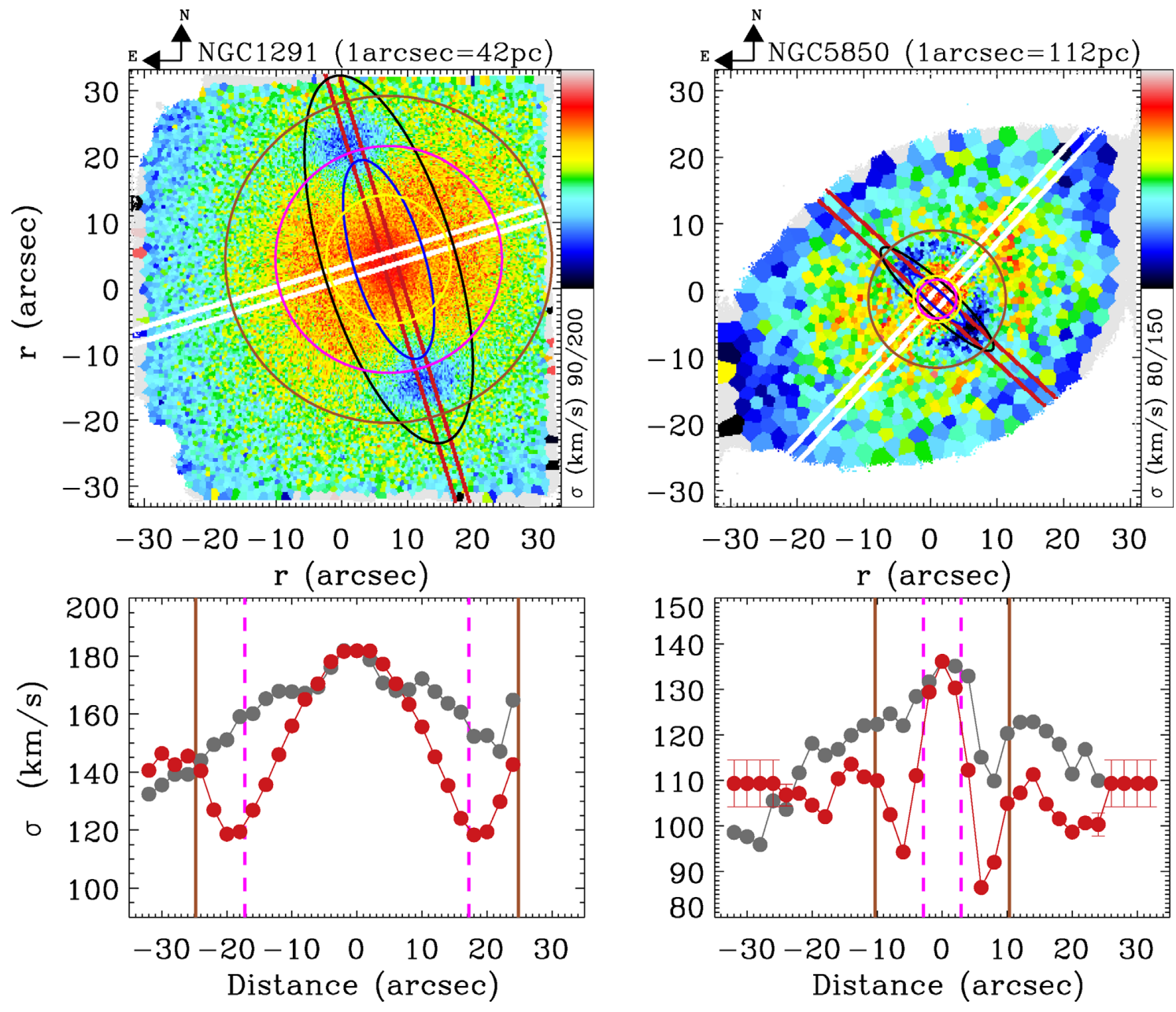

Figure 9. Analysis of the 2D spatial distribution of the stellar velocity dispersion for NGC 1291 (left) and NGC 5850 (right). The top panels show the stellar velocity dispersion maps; the projected size and shape of the bulges (as measured with their effective radii; yellow lines) and inner bars [as measured with both the full length (black lines) and the effective radii of the corresponding Ferrers profiles (blue lines)] are overplotted. Two circles indicating the radii at which the surface brightness of both the bulge and the inner bar are the same are also overplotted (magenta: inner crossing; brown: outer crossing). The $\sigma$-hollows appear within the region where the inner bar dominates over the bulge light. The red and white lines highlight the regions used as pseudo-slits to retrieve the linear $\sigma$ profiles along and perpendicular to the inner bars, respectively. These profiles (red and grey, respectively) are shown in the bottom panels, where the radii at which the surface brightness profiles of bulges and inner bars cross are also indicated (dashed magenta line: inner crossing; solid brown line: outer crossing).

disc, outer bar, and lens (Fig. 6). Fig. 10 shows the mean luminosityweighted ages, metallicities, and $[\alpha / \mathrm{Fe}]$ linearly averaged within the regions of predominance of each individual structure. It is important to remark here that although each component is dominant inside its own segment, its contribution cannot be fully isolated with respect to the rest of the overlapping structures such as, at least, the galaxy disc.

The errors associated with the mean values shown in Fig. 10 have been computed as the quadratic sum of the uncertainties in the age and metallicity measurements (typically of $0.9 \mathrm{Gyr}$ and 0.06 dex, respectively) and the standard deviation of the values that are averaged for each structure, corrected by the number of elements involved in the average.

The inside-out gradient already pointed out in Section 5.3 is clearly seen in Fig. 10 not only in age but also in metallicity and $[\alpha / \mathrm{Fe}]$. The centre (represented by the central PSF and the inner bulge) is the oldest, more metal-rich, and less $\alpha$-enhanced part of the galaxy. The remaining structures (outer bulge, inner bar, inner disc, outer bar, and lens) show old and particularly similar ages:
Values expand from 6.4 to $6.9 \mathrm{Gyr}$, and the metallicity and $[\alpha / \mathrm{Fe}]$ cover short ranges. This result points towards a rapid consumption of most of the gas available for triggering significant star formation, so most of the stars, now distributed along the whole galaxy, were formed at an early stage.

So far we have discussed when the stars in NGC 1291 were formed. In the following we will discuss how the morphological assembly of this galaxy took place. We emphasize here that the assembly of structures may or may not involve star formation and therefore the analysis of SFH in these regions does not necessarily imply the formation history of the structures. However, the combination of the SFH and the kinematic and structural analysis presented here is a powerful approach to constrain the assembly history of NGC 1291.

Within scenario 2, the bar structures in NGC 1291 were born from dynamical instabilities of the outer and inner disc. The inner disc was secularly formed after gas inflow along the outer bar. This process does involve star formation: The fact that the inner disc has the same age as the outer galaxy regions (outer bar and lens) 

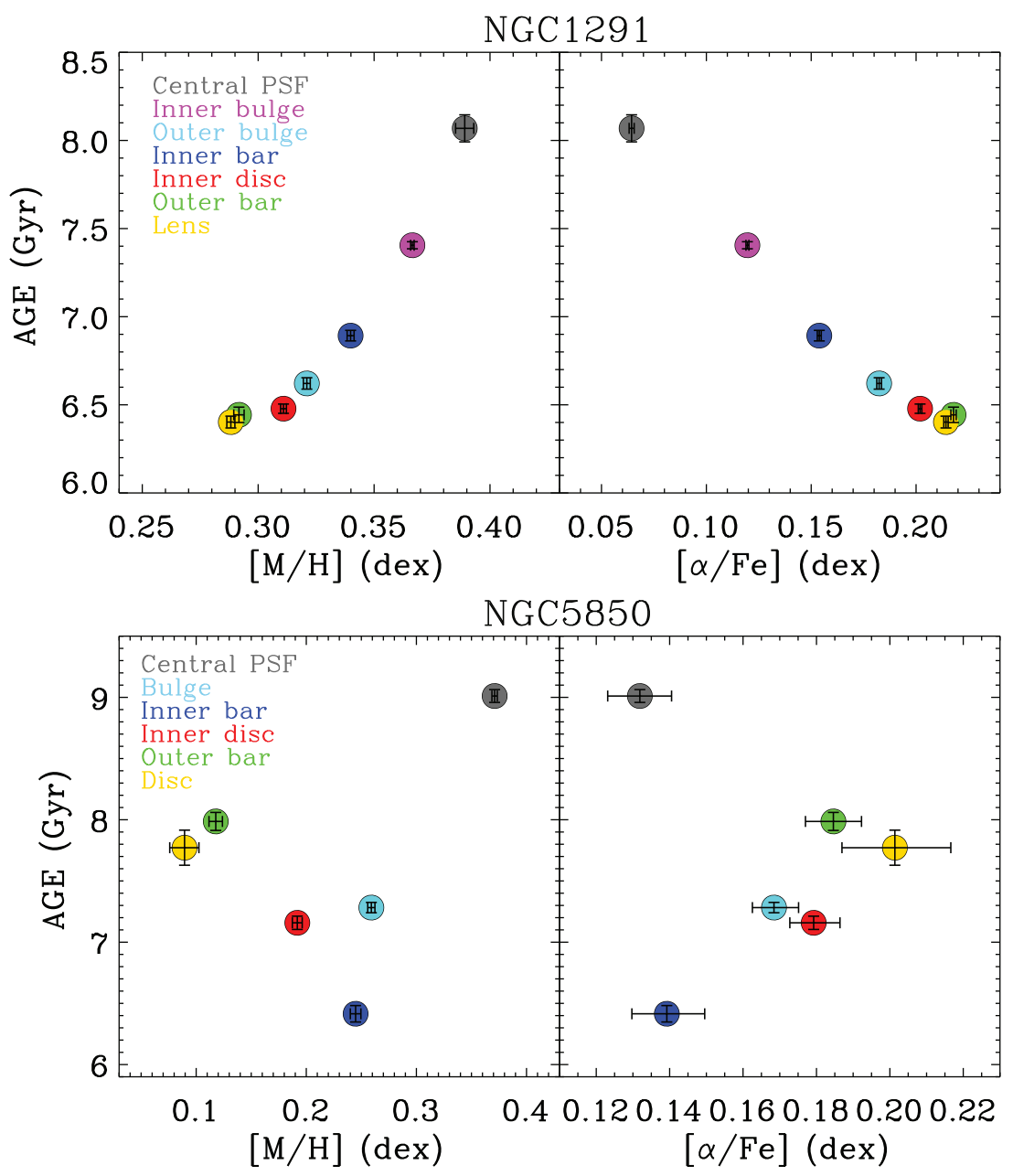

Figure 10. Mean luminosity-weighted age versus metallicity (left-hand panels) and $[\alpha / \mathrm{Fe}]$ (right-hand panels) within the spatial regions where each structural component of NGC 1291 (top) and NGC 5850 (bottom) dominates the light: inner bulge (purple; only for NGC 1291), outer bulge (light blue), inner disc (red), inner bar (blue), outer bar (green), and lens (NGC 5850)/galaxy disc (NGC 1291; yellow). Due to possible resolution effects, the central PSF is considered separately (grey). See Section 5.2 for details on the selection of these regions.

indicates that the whole process of outer bar formation, gas inflow, and inner disc formation was fast. This explains that no rejuvenation of the inner disc with respect to the outer bar is noticeable but the inner disc actually is slightly more metal-rich and $\alpha$-enhanced, as expected.

Once the inner disc had formed, the dynamical assembly of the inner bar happened at a later stage from the same stellar content. The fact that the inner bar is older than the inner disc is explained by invoking some level of star formation happening in the inner disc (as well as in the outer regions) after the inner bar was already assembled (as it has actually been seen in simulations and observations; Athanassoula 1992; Emsellem et al. 2015; Romeo \& Fathi 2016). This is not surprising: The shear exerted by the inner bar prevents star formation inside it, while the gas still present in the galaxy may continue forming stars outside.

The latest result also agrees with the fact that the outer bulge region, which is purposely taken where the contribution from the inner bar is negligible, is also younger than the inner bar. Only structures inside the inner bar remain the oldest, as the shear introduced by the inner bar prevents star formation and subsequent rejuvenation. We can also pose constraints on the moment of assembly of the inner bar: It had to take place before the slight rejuvenation of the inner disc and outer structures happened, i.e. $>6.5$ Gyr ago.

Important conclusions are inferred from this assembly history, as it implies this inner bar is a long-lived structure. This conclusion is further reinforced by the presence of a box/peanut associated with the inner bar of NGC 1291, as it will be explained in Section 6.3 (see also Méndez-Abreu et al. 2019). The transient versus long-living nature of inner bars has been a matter of debate for a long time since many numerical simulations, mostly those corresponding to scenario 1 , generate fragile inner bars that live for very short periods of time (dissolving in a few hundreds of Myr).

The old and metal-rich populations at the centre of NGC 1291 (central PSF, inner bulge) need to be explained through an early formation of these very central structures either in a very intense way with a high star formation rate which rapidly enriched the medium or from an already enriched reservoir of gas everywhere in the galaxy. This second option implies that an external supply of less metal-rich gas has to be invoked to explain the fuel for forming the rest of the components. We remind the reader that NGC 1291 belongs to a group (Huchra \& Geller 1982), but gas inflow from the galaxy halo can also explain these results. 


\subsubsection{NGC 5850: an inner bar assembled more than 4.5 Gyr ago}

The inner and outer structures within NGC 5850 are clearly distinguishable in their stellar populations. In this case, the inner bar is younger, more metal-rich, and less $\alpha$-enhanced than the outer bar. This result matches with predictions from scenario 1, as already pointed out in de Lorenzo-Cáceres et al. (2013). However, it also fits within scenario 2 and this second possibility is reinforced by the presence of the inner disc.

The results for NGC 5850 indicate its inner bar has a major contribution from stars between 1 and 4.5 Gyr old. Within scenario 2, this inner bar was dynamically assembled from the inner disc, but this is older, less metallic, and more $\alpha$-enhanced. These results can be easily reconciled if star formation occurred along the inner bar after it was assembled from the inner disc; such a star-forming period took place between 4.5 and $1 \mathrm{Gyr}$ ago. This is not surprising for an inner bar as strong as that of NGC 5850 (the inner bar in NGC 5850 shows $\varepsilon \sim 0.8$ and the ellipticity is a proxy for the bar strength, as indicated by e.g. Laurikainen, Salo \& Rautiainen 2002). When such a strong bar is formed, gas quickly reacts to its potential and star formation is triggered inside the barred structure (e.g. Elmegreen \& Elmegreen 1985; Friedli \& Benz 1995; Martin \& Friedli 1997). Such behaviour is not expected in NGC 1291, which hosts a weaker inner bar $(\varepsilon \sim 0.6)$ and therefore its shear produces the opposite effect of preventing star formation.

Since the rejuvenation of the inner bar with respect to the inner disc had to happen after the inner bar was dynamically assembled, the evidence shown in Fig. 7 indicates that it was fully formed $>4.5$ Gyr ago. As in NGC 1291, this result supports a long-living nature for the inner bar of NGC 5850. We note here that de LorenzoCáceres et al. $(2012,2013)$ also suggested inner bars are long-lived structures when studying four additional double-barred galaxies. To the best of our knowledge, no observational evidence of short-lived bars has been found so far.

As expected, the outer regions (outer bar and disc) of NGC 5850 are old, with a slight difference in age, so the outer bar is slightly older than the disc. As discussed for NGC 1291, this evidence may indicate that star formation was inhibited within the bar region once it had formed, while it continues happening within the disc. NGC 5850 indeed hosts a galaxy disc with spiral arms where star formation is noticeable. We emphasize, however, that the MUSE FoV probes the NGC 5850 innermost disc regions, at the same galactic longitudes where the bar exists.

The central PSF of NGC 5850, a galaxy in a pair (Madore, Freedman \& Bothun 2004), shows the same very old, more metalrich, and less $\alpha$-enhanced behaviour as NGC 1291. The bulge in this case corresponds to a region where the contribution from the inner bar is negligible (like the outer bulge in NGC 1291) and it shows stellar-population properties closer to those of the inner disc. Such very old and metal-rich central regions are found for many TIMER galaxies and will be discussed in a forthcoming paper (SánchezBlázquez et al., in preparation).

\subsection{Secular evolution driven by inner bars}

Bars are considered the main drivers of secular evolution as, thanks to their non-axisymmetric potential, they can promote stellar radial migration outwards and gas inflow, which eventually may trigger star formation. Inner bars are also considered capable of such an effect. They have even been proposed as a mechanism to bring gas closer to the sphere of influence of the supermassive black holes (Shlosman et al. 1989, 1990); gas transported by a single bar cannot reach distances less than $\sim 100 \mathrm{pc}$ from the centre. This idea of secular evolution promoted by inner bars is further supported by scenario 2, which implies that all bars have the same nature. However, no evidence of any rejuvenation due to gas inflow through inner bars is found in these galaxies; in de Lorenzo-Cáceres et al. $(2012,2013)$ similar results were found, as well as signatures of gas inflow from the outer to the inner bars. Gas may indeed be inflowing but not forming stars in a significant way. Analysis of the ionized gas in the TIMER double-barred galaxies will be presented in a forthcoming paper.

Numerical simulations have shown that buckling instabilities in bars are responsible for the formation of the so-called box/peanut structures at galaxy centres (e.g. Martinez-Valpuesta, Shlosman \& Heller 2006, and references therein). This secular process promoted by bars is based on the redistribution of existing stars and does not imply new star formation; i.e. different stellar population content for the box/peanut with respect to the bar is not expected. Whether inner bars may drive box/peanut formation as well has not been assessed in the literature so far. In a companion paper (Méndez-Abreu et al. 2019) we demonstrate that the inner bar of NGC 1291 does show the expected kinematic signatures of a box/peanut seen face-on in its $h_{4}$ distribution (see Debattista et al. 2005 and Méndez-Abreu et al. $2008 \mathrm{~b}$ for numerical and observational evidence, respectively).

Estimates from numerical simulations indicate that box/peanuts are typically born $\sim 1$ Gyr after the formation of the bar. Whether this time-scale holds for small-scale bars has not been studied yet and is debatable. If so, this would be a further confirmation of the long-lived nature of inner bars. Finally, the presence of a box/peanut structure, so far associated with large-scale bars alone, reinforces the idea of inner and outer bars having the same nature. This conclusion once again matches with the hypothesis of scenario 2 . We refer the reader to Méndez-Abreu et al. (2019) for more discussion on these topics.

The nature of the bulge in NGC 5850 is not so clear as in NGC 1291. The bulge component has a Sérsic index of 2.68 as derived from the photometric decompositions presented in Section 3. This would correspond to a classical bulge under the criterion of Fisher \& Drory (2008), but the use of this photometric diagnostic for retrieving the bulge nature has recently been challenged by several authors (e.g. Costantin et al. 2017; Méndez-Abreu et al. 2018). In any case and as discussed before, we do not find any evidence of recent star formation bursts thanks to gas inflow along the inner bar. What we do find is that the central region (central PSF in the plots) does show lower $[\alpha / \mathrm{Fe}]$ values, pointing towards a more extended star formation process; intriguingly, it also has the oldest stellar populations.

\section{SUMMARY AND CONCLUSIONS}

TIMER is a project devoted to the detailed study (gas and stellar content) of 24 barred galaxies hosting presumably secularly formed inner structures that have been observed with the MUSE integralfield spectrograph. In this paper, we present the analysis of the two double-barred galaxies included in the TIMER sample: NGC 1291 and NGC 5850. Given the structural complexity of double-barred systems, we have first performed 2D multicomponent photometric decompositions of the $S^{4} \mathrm{G}$ images for these galaxies, in order to disentangle the mix of structures lying at the central regions covered by the MUSE FoV. A careful analysis of the stellar properties (kinematics and stellar populations) of these structures is carried out throughout the paper. The main observational results obtained are as follows. 
(i) Both galaxies host a stellar inner disc matching in size with the corresponding inner bar. While the inner disc in NGC 5850 had already been noticed through kinematical analysis, the one in NGC 1291 (a face-on galaxy) has been unveiled by our 2D photometric decomposition.

(ii) The presence of $\sigma$-hollows, two local decreases of the stellar velocity dispersion values at the ends of inner bars (de LorenzoCáceres et al. 2008), is confirmed for the two double-barred galaxies analysed here.

(iii) NGC 5850 shows a ring-like feature with high $h_{4}$ values as expected for inner bars (Du et al. 2016). The $h_{4}$ distribution for NGC 1291 is more complicated and it is the subject of a companion paper (Méndez-Abreu et al. 2019).

(iv) Inner bars show clearly distinct stellar populations than the surrounding regions. In NGC 5850, the inner bar is younger and more metal-rich, confirming the results by de Lorenzo-Cáceres et al. (2013). On the contrary, NGC 1291 hosts an older and more metalrich inner bar than the outer structures (inner disc, outer bar, lens, and even the outermost part of the bulge, outside the inner bar region).

(v) The stellar age distribution and age-metallicity relation for the dominant structural components of NGC 1291 within the MUSE FoV, namely the bulge, inner bar, inner disc, outer bar, and lens, are rather similar.

(vi) The star formation history for the inner bar of NGC 5850 shows a significant bump between 1 and $4.5 \mathrm{Gyr}$. All other structures (bulge, inner disc, outer bar, and disc) have smoothly declining profiles.

(vii) Both inner bars show lower $[\alpha / \mathrm{Fe}]$ values than the outer regions.

The above observational pieces of evidence have been discussed within the frameworks of the two main formation scenarios proposed for inner bars: scenario 1, in which the inner bar is directly assembled through a gas-rich process that implies in situ star formation in the inner bar (e.g. Friedli \& Martinet 1993) - according to simulations, this scenario usually forms fragile inner bars - and scenario 2 , in which the origin of inner bars is exactly the same as that for outer bars, i.e. formation through dynamical instabilities in cold discs, but at smaller spatial scales (e.g. Debattista \& Shen 2007). This scenario has been successful in simulating longlived inner bars. The main conclusions derived from the analysis presented here regarding the formation and evolution of inner bars are as follows.

(i) Given the presence of inner discs in the majority of doublebarred galaxies studied in detail (see also the works by de LorenzoCáceres et al. 2012, 2013), and the results obtained for NGC 1291 (inner bar appears slightly older than outer bar), we suggest scenario 2 is better suited to explain how double-barred galaxies form.

(ii) The fact that all structures in NGC 1291 present similar old ages and metallicities suggest this galaxy formed all stars and assembled all components (as the inner disc is most probably the result of a star-forming process) within a short time-scale.

(iii) The rejuvenation of the inner disc in NGC 1291 with respect to the inner bar may be due to a slightly longer star formation process in the inner disc after the inner bar was formed. The star formation in the weak $(\varepsilon \sim 0.6)$ inner bar would thus have been quenched when it was assembled, as predicted by Athanassoula (1992) and Emsellem et al. (2015) for large-scale bars. This result constrains the moment of assembly of the inner bar to an early epoch, so its mean age is older than $6.5 \mathrm{Gyr}$. (iv) NGC 5850 hosts a strong inner bar $(\varepsilon \sim 0.8)$ for which star formation triggering right after inner-bar assembly is expected, as predicted by e.g. Friedli \& Benz (1995). This rejuvenation process, further supported by the younger and more metal-rich stellar content of this inner bar, took place between 1 and $4.5 \mathrm{Gyr}$ ago. Therefore, the inner bar was assembled before it could be rejuvenated with respect to the inner disc, i.e. $\sim 4.5 \mathrm{Gyr}$ ago.

(v) The assembly epochs for the inner bars derived in this work and the fact that the inner bar in NGC 1291 has developed a box/peanut structure (Méndez-Abreu et al. 2019) backs the hypothesis that inner bars are long-lived structures, as suggested by several numerical works (e.g. Wozniak 2015).

(vi) We find no significantly younger stellar populations for the bulges with respect to the inner bars, nor signatures of more recent star formation after the inner disc formation that could be due to secular evolution promoted by inner bars.

In summary, by combining a structural analysis with a star formation history analysis and thanks to the superb properties of the TIMER MUSE data, we have for the first time clocked the dynamical assembly history of double-barred galaxies, which supports the facts that (i) inner bars are dynamically formed from discs in an analogous way as outer bars and (ii) inner bars are long-lived structures.

\section{ACKNOWLEDGEMENTS}

We are grateful to Peter Erwin for providing his HST data for NGC 4984 for inspection, as well as to the anonymous referee for many valuable comments which helped improving this manuscript. AdLC and JMA thank the European Southern Observatory for the warm hospitality during a science visit when part of this work was done. This work is based on observations collected at the European Organisation for Astronomical Research in the Southern Hemisphere under ESO program 097.B-0640(A). AdLC acknowledges support from grant AYA2016-77237-C3-1-P from the Spanish Ministry of Economy and Competitiveness (MINECO). PSB acknowledges support from grant AYA2016-77237-C3-2$\mathrm{P}$ from the Spanish Ministry of Economy and Competitiveness (MINECO). JMA acknowledges support from the Spanish Ministry of Economy and Competitiveness (MINECO) by grants AYA201343188-P and AYA2017-83204-P. GvdV acknowledges funding from the European Research Council (ERC) under the European Union's Horizon 2020 research and innovation programme under grant agreement No 724857 (Consolidator Grant ArcheoDyn).

\section{REFERENCES}

Aguerri J. A. L., Méndez-Abreu J., Corsini E. M., 2009, A\&A, 495, 491

Athanassoula E., 1992, MNRAS, 259, 345

Athanassoula E., 2003, MNRAS, 341, 1179

Athanassoula E., Morin S., Wozniak H., Puy D., Pierce M. J., Lombard J., Bosma A., 1990, MNRAS, 245, 130

Bureau M., Athanassoula E., 2005, ApJ, 626, 159

Burstein D., Faber S. M., Gaskell C. M., Krumm N., 1984, ApJ, 287, 586

Buta R. J. et al., 2015, ApJS, 217, 32

Cappellari M., Emsellem E., 2004, PASP, 116, 138

Cervantes J. L., Vazdekis A., 2009, MNRAS, 392, 691

Combes F., Debbasch F., Friedli D., Pfenniger D., 1990, A\&A, 233, 82

Corsini E. M., Debattista V. P., Aguerri J. A. L., 2003, ApJ, 599, L29

Costantin L., Méndez-Abreu J., Corsini E. M., Morelli L., Aguerri J. A. L., Dalla Bontà E., Pizzella A., 2017, A\&A, 601, A84 
de Lorenzo-Cáceres A., Falcón-Barroso J., Vazdekis A., Martínez-Valpuesta I., 2008, ApJ, 684, L83

de Lorenzo-Cáceres A., Vazdekis A., Aguerri J. A. L., Corsini E. M., Debattista V. P., 2012, MNRAS, 420, 1092

de Lorenzo-Cáceres A., Falcón-Barroso J., Vazdekis A., 2013, MNRAS, 431, 2397

de Lorenzo-Cáceres A., Méndez-Abreu J., Thorne B., Costantin L., 2019a, MNRAS, 484, 665

de Lorenzo-Cáceres A., Mendez-Abreu J., Thorne B., Costantin L., 2019b, MNRAS, preprint (arXiv:1901.08881)

Debattista V. P., Sellwood J. A., 2000, ApJ, 543, 704

Debattista V. P., Shen J., 2007, ApJ, 654, L127

Debattista V. P., Carollo C. M., Mayer L., Moore B., 2005, ApJ, 628, 678

Du M., Shen J., Debattista V. P., 2015, ApJ, 804, 139

Du M., Debattista V. P., Shen J., Cappellari M., 2016, ApJ, 828, 14

Du M., Shen J., Debattista V. P., de Lorenzo-Cáceres A., 2017, ApJ, 836, 181

Elmegreen B. G., Elmegreen D. M., 1985, ApJ, 288, 438

Emsellem E., Greusard D., Combes F., Friedli D., Leon S., Pécontal E., Wozniak H., 2001, A\&A, 368, 52

Emsellem E., Renaud F., Bournaud F., Elmegreen B., Combes F., Gabor J. M., 2015, MNRAS, 446, 2468

Englmaier P., Shlosman I., 2004, ApJ, 617, L115

Erwin P., 2004, A\&A, 415, 941

Erwin P., 2005, MNRAS, 364, 283

Erwin P., 2011, Mem. Soc. Astron. Ital. Suppl., 18, 145

Erwin P., Sparke L. S., 2002, AJ, 124, 65

Falcón-Barroso J., Sánchez-Blázquez P., Vazdekis A., Ricciardelli E., Cardiel N., Cenarro A. J., Gorgas J., Peletier R. F., 2011, A\&A, 532, A95

Ferrers N. M., 1877, Quart. J. Pure and Appl. Math, 14, 1

Fisher D. B., Drory N., 2008, AJ, 136, 773

Freeman K. C., 1970, ApJ, 160, 811

Friedli D., Benz W., 1995, A\&A, 301, 649

Friedli D., Martinet L., 1993, A\&A, 277, 27

Gadotti D. A., Seidel M. K., Sánchez-Blázquez P., Falcón-Barroso J., Husemann B., Coelho P., Pérez I., 2015, A\&A, 584, A90

Gadotti D. A. et al., 2019, MNRAS, 482, 506 (Paper I)

Gerhard O. E., 1993, MNRAS, 265, 213

Heller C., Shlosman I., Englmaier P., 2001, ApJ, 553, 661

Huchra J. P., Geller M. J., 1982, ApJ, 257, 423

Koleva M., Bavouzet N., Chilingarian I., Prugniel P., 2007, in KisslerPatig M., Walsh J. R., Roth M. M., eds, Science Perspectives for 3D Spectroscopy Validation of Stellar Population and Kinematical Analysis of Galaxies. Springer-Verlag, Berlin, Heidelberg, p. 153

Krajnović D. et al., 2008, MNRAS, 390, 93

Kroupa P., 2001, MNRAS, 322, 231

Laine S., Shlosman I., Knapen J. H., Peletier R. F., 2002, ApJ, 567, 97

Laurikainen E., Salo H., Rautiainen P., 2002, MNRAS, 331, 880

Lisker T., Debattista V. P., Ferreras I., Erwin P., 2006, MNRAS, 370, 477

Madore B. F., Freedman W. L., Bothun G. D., 2004, ApJ, 607, 810

Marinova I., Jogee S., 2007, ApJ, 659, 1176

Martin P., Friedli D., 1997, A\&A, 326, 449
Martín-Navarro I., Vazdekis A., Falcón-Barroso J., La Barbera F., Yıldırım A., van de Ven G., 2018, MNRAS, 475, 3700

Martinez-Valpuesta I., Shlosman I., Heller C., 2006, ApJ, 637, 214

Méndez-Abreu J. et al., 2019, MNRAS, 482, L118

Méndez-Abreu J., Aguerri J. A. L., Corsini E. M., Simonneau E., 2008a, A\&A, 478, 353

Méndez-Abreu J., Corsini E. M., Debattista V. P., De Rijcke S., Aguerri J. A. L., Pizzella A., 2008b, ApJ, 679, L73

Méndez-Abreu J., Sánchez-Janssen R., Aguerri J. A. L., Corsini E. M., Zarattini S., 2012, ApJ, 761, L6

Méndez-Abreu J., Debattista V. P., Corsini E. M., Aguerri J. A. L., 2014, A\&A, 572, A25

Méndez-Abreu J. et al., 2017, A\&A, 598, A32

Méndez-Abreu J. et al., 2018, MNRAS, 474, 1307

Moiseev A. V., Valdés J. R., Chavushyan V. H., 2004, A\&A, 421, 433

Muñoz-Tuñón C., Caon N., Aguerri J. A. L., 2004, AJ, 127, 58

Ocvirk P., Pichon C., Lançon A., Thiébaut E., 2006a, MNRAS, 365, 74

Ocvirk P., Pichon C., Lançon A., Thiébaut E., 2006b, MNRAS, 365, 46

Petitpas G. R., Wilson C. D., 2002, ApJ, 575, 814

Petitpas G. R., Wilson C. D., 2004, ApJ, 603, 495

Pietrinferni A., Cassisi S., Salaris M., Hidalgo S., 2013, A\&A, 558, A46

Rautiainen P., Salo H., 2000, A\&A, 362, 465

Rautiainen P., Salo H., Laurikainen E., 2002, MNRAS, 337, 1233

Romeo A. B., Fathi K., 2016, MNRAS, 460, 2360

Sánchez-Blázquez P. et al., 2006, MNRAS, 371, 703

Sánchez-Blázquez P., Ocvirk P., Gibson B. K., Pérez I., Peletier R. F., 2011, MNRAS, 415, 709

Sarzi M. et al., 2006, MNRAS, 366, 1151

Sérsic J. L., 1968, Atlas de Galaxias Australes. Observatorio Astronomico, Cordoba, Argentina

Shen J., Debattista V. P., 2009, ApJ, 690, 758

Sheth K., Vogel S. N., Regan M. W., Thornley M. D., Teuben P. J., 2005 ApJ, 632, 217

Sheth K. et al., 2010, PASP, 122, 1397

Shlosman I., Heller C. H., 2002, ApJ, 565, 921

Shlosman I., Frank J., Begelman M. C., 1989, Nature, 338, 45

Shlosman I., Begelman M. C., Frank J., 1990, Nature, 345, 679

van der Marel R. P., Franx M., 1993, ApJ, 407, 525

Vazdekis A., Ricciardelli E., Cenarro A. J., Rivero-González J. G., DíazGarcía L. A., Falcón-Barroso J., 2012, MNRAS, 424, 157

Vazdekis A. et al., 2015, MNRAS, 449, 1177

Vazdekis A., Koleva M., Ricciardelli E., Röck B., Falcón-Barroso J., 2016, MNRAS, 463, 3409

Weilbacher P. M., Streicher O., Urrutia T., Jarno A., Pécontal-Rousset A., Bacon R., Böhm P., 2012, in Proc. SPIE Conf. Ser. Vol. 8451, Design and Capabilities of the MUSE Data Reduction Software and Pipeline. SPIE, Bellingham, p. 84510B

Worthey G., Faber S. M., González J. J., Burstein D., 1994, ApJS, 94, 687

Wozniak H., 2015, A\&A, 575, A7

This paper has been typeset from a $\mathrm{T}_{\mathrm{E}} \mathrm{X} / \mathrm{L} \mathrm{T}_{\mathrm{E}} \mathrm{X}$ file prepared by the author. 